\title{
A Finite Difference Method for Studying Thermal Deformation in 3D Double-Layered Micro-Structures Exposed to Ultrashort-Pulsed Lasers
}

\author{
Suyang Zhang, Pan Wang and Weizhong Dai*
}

\author{
Mathematics \& Statistics, College of Engineering \& Science, Louisiana Tech University, Ruston, LA 71272, USA
}

\begin{abstract}
Ultrashort-pulsed lasers have been attracting worldwide interest in science and engineering. Studying the thermal deformation induced by ultrashort-pulsed lasers is important for preventing thermal damage. This article presents a new numerical method for studying thermal deformation in 3D double-layered film micro-structures exposed to ultrashort-pulsed lasers. The method is demonstrated by investigating thermal deformations in a 3D double-layered thin film and a 3D double-layered sphere, respectively.
\end{abstract}

\section{INTRODUCTION}

Ultrashort-pulsed lasers have been attracting worldwide interest in science and engineering, because their pulse durations are of the order of sub-picoseconds to femtoseconds and because they possess exclusive capabilities of limiting the undesirable spread of the thermal process zone in the heated sample [1]. The success of using high-energy ultrashort-pulsed lasers in real applications relies on three factors [1]: (1) well characterized pulse width, intensity and experimental techniques; (2) reliable microscale heat transfer models; and (3) prevention of thermal damage. Up to date, a number of models that focus on heat transfer in the context of ultrashort-pulsed lasers have been developed. However, only a few mathematical models for studying thermal deformation induced by ultrashort-pulsed lasers have been developed [1-7]. Tzou and his colleagues [1] presented a onedimensional model in a double-layered thin film. The model was solved using a differential-difference approach. Chen and his colleagues [5] considered a two-dimensional axisymmetric cylindrical thin film and proposed an explicit finite difference method by adding an artificial viscosity term to eliminate numerical oscillations. Dai and his colleagues $[2,4]$ developed a new method for studying thermal deformation in 2D thin films exposed to ultrashort-pulsed lasers. The method was obtained based on the parabolic two-step heat transport equations and implicit finite difference schemes on a staggered grid. It accounts for the coupling effect between lattice temperature and strain rate, as well as for the hot-electron blast effect in momentum transfer. Numerical results show that there are no numerical oscillations in the solution. Unfortunately, when applied to a 3D thin film case, they found that the nonphysical oscillations appeared again in the normal stress in the thickness direction. Recently, Dai and his colleagues $[8,9]$ have improved their previous method by developing a fourth order compact finite difference scheme for solving the dynamic equations of motion. Results show that the non-physical oscillations disappear. In this article, we extend this method to study thermal deformation

*Address correspondence to this author at the Mathematics \& Statistics, College of Engineering \& Science, Louisiana Tech University, Ruston, LA 71272, USA; Tel: 1-318-257-3301; Fax: 1-318-257-2562;

E-mail: dai@coes.latech.edu in 3D double-layered metal thin films and micro spheres exposed to ultrashort-pulsed lasers. Layered metal thin films are considered because they are widely used in engineering applications due to the fact that a single metal layer often cannot satisfy all mechanical, thermal and electronic requirements, while micro spheres are of interest related to micro resonators in optical applications, such as ultra-lowthreshold lasing, sensing, optoelectronic microdevices, cavity quantum electrodynamics and their potential in quantum information processing. This research provides a numerical method for studying thermal deformations induced by ultrashort-pulsed lasers when layered micro-structures are considered.

\section{MATHMATICAL MODEL}

Consider a 3D double-layered thin film in Cartesian coordinates, which is exposed to an ultrashort-pulsed laser, as shown in Fig. (1a). The governing equations for studying thermal deformation in the thin film can be expressed as follows:

(1) Dynamic Equations of Motion [1, 2, 5, 9]

$$
\begin{aligned}
\rho^{(m)} \frac{\partial^{2} u^{(m)}}{\partial t^{2}} & =\frac{\partial \sigma_{x}^{(m)}}{\partial x}+\frac{\partial \sigma_{x y}^{(m)}}{\partial y}+\frac{\partial \sigma_{x z}^{(m)}}{\partial z} \\
& +2 \Lambda^{(m)} T_{e}^{(m)} \frac{\partial T_{e}^{(m)}}{\partial x}, \\
\rho^{(m)} \frac{\partial^{2} v^{(m)}}{\partial t^{2}} & =\frac{\partial \sigma_{x y}^{(m)}}{\partial x}+\frac{\partial \sigma_{y}^{(m)}}{\partial y}+\frac{\partial \sigma_{y z}^{(m)}}{\partial z} \\
& +2 \Lambda^{(m)} T_{e}^{(m)} \frac{\partial T_{e}^{(m)}}{\partial y}, \\
\rho^{(m)} \frac{\partial^{2} w^{(m)}}{\partial t^{2}} & =\frac{\partial \sigma_{x z}^{(m)}}{\partial x}+\frac{\partial \sigma_{y z}^{(m)}}{\partial y}+\frac{\partial \sigma_{z}^{(m)}}{\partial z} \\
& +2 \Lambda^{(m)} T_{e}^{(m)} \frac{\partial T_{e}^{(m)}}{\partial z},
\end{aligned}
$$

where

$$
\begin{aligned}
\sigma_{x}^{(m)} & =\lambda^{(m)}\left(\varepsilon_{x}^{(m)}+\varepsilon_{y}^{(m)}+\varepsilon_{z}^{(m)}\right)+2 \mu^{(m)} \varepsilon_{x}^{(m)} \\
& -\left(3 \lambda^{(m)}+2 \mu^{(m)}\right) \alpha_{T}^{(m)}\left(T_{l}^{(m)}-T_{0}\right),
\end{aligned}
$$




\section{Ultrashort-pulsed lasers}

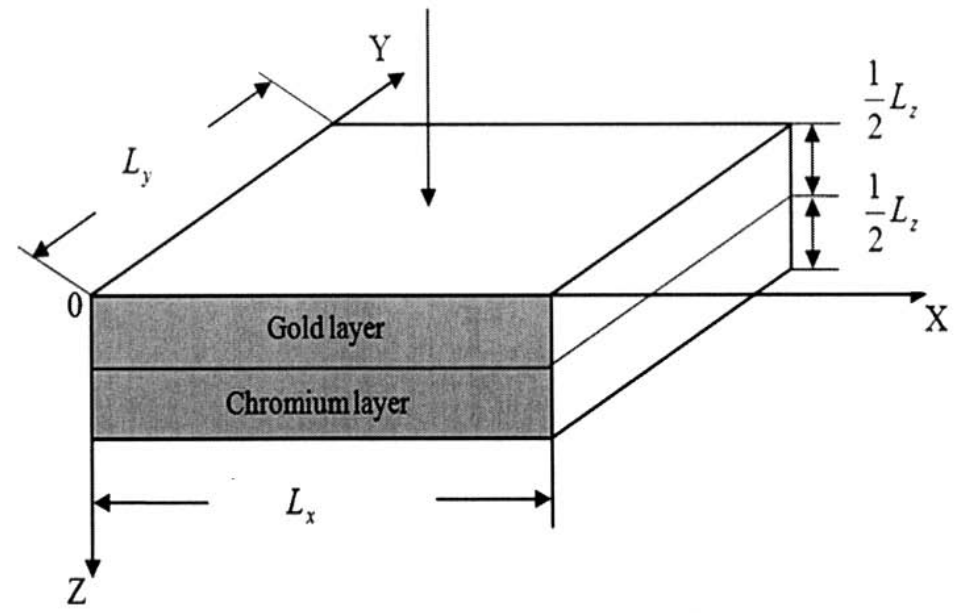

(a)

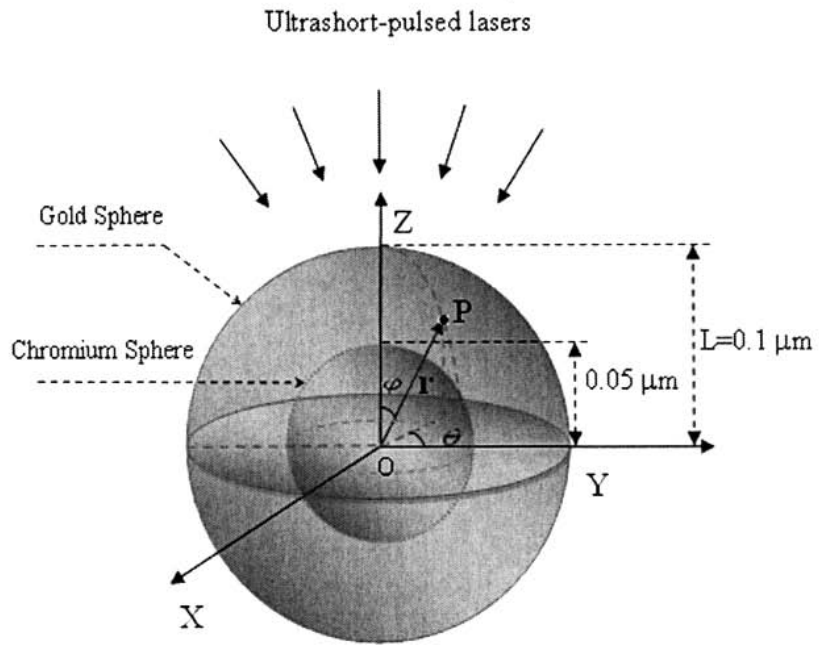

(b)

Fig. (1a). A 3D thin film and (b) a 3D micro sphere irradiated by ultrashort-pulsed lasers.

$$
\begin{aligned}
\sigma_{y}^{(m)} & =\lambda^{(m)}\left(\varepsilon_{x}^{(m)}+\varepsilon_{y}^{(m)}+\varepsilon_{z}^{(m)}\right)+2 \mu^{(m)} \varepsilon_{y}^{(m)} \\
& -\left(3 \lambda^{(m)}+2 \mu^{(m)}\right) \alpha_{T}^{(m)}\left(T_{l}^{(m)}-T_{0}\right), \\
\sigma_{z}^{(m)} & =\lambda^{(m)}\left(\varepsilon_{x}^{(m)}+\varepsilon_{y}^{(m)}+\varepsilon_{z}^{(m)}\right)+2 \mu^{(m)} \varepsilon_{z}^{(m)} \\
& -\left(3 \lambda^{(m)}+2 \mu^{(m)}\right) \alpha_{T}^{(m)}\left(T_{l}^{(m)}-T_{0}\right), \\
\sigma_{x y}^{(m)} & =\mu^{(m)} \gamma_{x y}^{(m)}, \sigma_{x z}^{(m)}=\mu^{(m)} \gamma_{x z}^{(m)}, \\
\sigma_{y z}^{(m)} & =\mu^{(m)} \gamma_{y z}^{(m)}, \\
\varepsilon_{x}^{(m)} & =\frac{\partial u^{(m)}}{\partial x}, \varepsilon_{y}^{(m)}=\frac{\partial v^{(m)}}{\partial y}, \varepsilon_{z}^{(m)}=\frac{\partial w^{(m)}}{\partial z}, \\
\gamma_{x y}^{(m)} & =\frac{\partial u^{(m)}}{\partial y}+\frac{\partial v^{(m)}}{\partial x}, \gamma_{x z}^{(m)}=\frac{\partial u^{(m)}}{\partial z}+\frac{\partial w^{(m)}}{\partial x}, \\
\gamma_{y z}^{(m)} & =\frac{\partial v^{(m)}}{\partial z}+\frac{\partial w^{(m)}}{\partial y} .
\end{aligned}
$$

Here, $m=1,2$, denotes layer 1 and layer 2, respectively; $u^{(m)}, v^{(m)}, w^{(m)}$ are the displacements

in the $x, y, z$ directions, respectively; $\varepsilon_{x}^{(m)}, \varepsilon_{y}^{(m)}$, and

$\varepsilon_{z}^{(m)}$ are the normal strains in the $x, y$ and $z$ directions, respectively; $\gamma_{x y}^{(m)}$ is the shear strain in the $x y$ plane, $\gamma_{x z}^{(m)}$ is the shear strain in the $x z$ plane, $\gamma_{y z}^{(m)}$ is the shear strain in the $y z$ plane; $\sigma_{x}^{(m)}, \sigma_{y}^{(m)}$ and $\sigma_{z}^{(m)}$ are the normal stresses in the $x$, $y$ and $z$ directions, respectively; $\sigma_{x y}^{(m)}$ is the shear stress in the $x y$ plane, $\sigma_{x z}^{(m)}$ is the shear stress in the $x z$ plane, $\sigma_{y z}^{(m)}$ is the shear stress in the $y z$ plane; $T_{e}^{(m)}$ and $T_{l}^{(m)}$ are electron and lattice temperatures, respectively; $T_{0}$ is the initial temperature; $\rho^{(m)}$ is density; $\Lambda^{(m)}$ is the electron-blast coefficient; 
$\lambda^{(m)} \quad\left(=K^{(m)}-\frac{2}{3} \mu^{(m)} \quad[10]\right)$ and $\mu^{(m)}$ are Lame's coefficients; and $\alpha_{T}^{(m)}$ is the thermal expansion coefficient.

(2) Energy Equations $[1,2,5,11]$

$$
\begin{aligned}
C_{e}^{(m)}\left(T_{e}\right) \frac{\partial T_{e}^{(m)}}{\partial t} & =\frac{\partial}{\partial x}\left(k_{e}^{(m)}\left(T_{e}, T_{l}\right) \frac{\partial T_{e}^{(m)}}{\partial x}\right) \\
& +\frac{\partial}{\partial y}\left(k_{e}^{(m)}\left(T_{e}, T_{l}\right) \frac{\partial T_{e}^{(m)}}{\partial y}\right) \\
& +\frac{\partial}{\partial z}\left(k_{e}^{(m)}\left(T_{e}, T_{l}\right) \frac{\partial T_{e}^{(m)}}{\partial z}\right) \\
& -G^{(m)}\left(T_{e}^{(m)}-T_{l}^{(m)}\right)+Q, \\
C_{l}^{(m)} \frac{\partial T_{l}^{(m)}}{\partial t}= & G^{(m)}\left(T_{e}^{(m)}-T_{l}^{(m)}\right) \\
& -\left(3 \lambda^{(m)}+2 \mu^{(m)}\right) \alpha_{T}^{(m)} T_{0} \\
& \times \frac{\partial}{\partial t}\left(\varepsilon_{x}^{(m)}+\varepsilon_{y}^{(m)}+\varepsilon_{Z}^{(m)}\right),
\end{aligned}
$$

where the heat source introduced by [5] is extended for a Gaussian laser beam focusing at $\left(x_{0}, y_{0}\right)$ on the top surface as

$$
\begin{aligned}
& Q(x, y, z, t) \\
& =0.94 J \frac{1-R}{t_{p} z_{s}} \exp \left(-\frac{z}{z_{s}}-\frac{\left(x-x_{0}\right)^{2}+\left(y-y_{0}\right)^{2}}{r_{s}^{2}}\right. \\
& \left.\quad-2.77\left(\frac{t-2 t_{p}}{t_{p}}\right)^{2}\right) .
\end{aligned}
$$

Here, $C_{e}^{(m)}\left(T_{e}\right)=C_{e 0}^{(m)}\left(\frac{T_{e}^{(m)}}{T_{0}}\right)$ is the electron heat capacity, $k_{e}^{(m)}\left(T_{e}, T_{l}\right)=k_{0}^{(m)}\left(\frac{T_{e}^{(m)}}{T_{l}^{(m)}}\right)$ is the thermal conductivity, $G^{(m)}$ is the electron-lattice coupling factor, $C_{l}^{(m)}$ is the lattice heat capacity, respectively; $Q$ is the energy absorption rate; $J$ is the laser fluence; $R$ is the surface reflectivity; $t_{p}$ is the laser pulse duration; $z_{s}$ is the optical penetration depth; $r_{s}$ is the spatial profile parameter. In addition, 0.94 and 2.77 in Eq. (7) are given in $[11,12]$. Eqs. (5) and (6) are often referred to as parabolic two-step heat transport equations [13]. It should be pointed out that the term $\left(3 \lambda^{(m)}+2 \mu^{(m)}\right) \alpha_{T}^{(m)} T_{0} \frac{\partial}{\partial t}\left(\varepsilon_{x}^{(m)}+\varepsilon_{y}^{(m)}+\varepsilon_{Z}^{(m)}\right)$ is added in Eq. (6) to consider the coupling effect between lattice temperature and strain rate. However, from our experience the strain rate effect is insignificant.

The boundary conditions are assumed to be stress free [1, 5] and no heat losses from the surface in the short time response [12]:

$\sigma_{x}^{(m)}=0, \sigma_{x y}^{(m)}=0, \sigma_{x z}^{(m)}=0$, at $x=0, L_{x}$, $\sigma_{y}^{(m)}=0, \sigma_{x y}^{(m)}=0, \sigma_{y z}^{(m)}=0$, at $y=0, L_{y}$,

$\sigma_{z}^{(m)}=0, \sigma_{x z}^{(m)}=0, \sigma_{y z}^{(m)}=0$, at $z=0, L_{z}$,

$\frac{\partial T_{e}^{(m)}}{\partial \vec{n}}=0, \frac{\partial T_{l}^{(m)}}{\partial \vec{n}}=0$,

where $\vec{n}$ is the unit outward normal vector on the boundary.

The interfacial conditions are assumed to be perfect thermal contact at $z=\frac{L_{z}}{2}$ (the continuity of temperature and heat flux across the interface),

$u^{(1)}=u^{(2)}, v^{(1)}=v^{(2)}, w^{(1)}=w^{(2)}$,

$\sigma_{z}^{(1)}=\sigma_{z}^{(2)}, \sigma_{x z}^{(1)}=\sigma_{x z}^{(2)}, \sigma_{y z}^{(1)}=\sigma_{y z}^{(2)}$,

$T_{e}^{(1)}=T_{e}^{(2)}, k_{e}^{(1)} \frac{\partial T_{e}^{(1)}}{\partial z}=k_{e}^{(2)} \frac{\partial T_{e}^{(2)}}{\partial z}$.

The initial conditions at $t=0$ are assumed to be

$T_{e}^{(m)}=T_{l}^{(m)}=T_{0}, u^{(m)}=v^{(m)}=w^{(m)}=0$,

$u_{t}^{(m)}=v_{t}^{(m)}=w_{t}^{(m)}=0$,

It should be pointed out that the laser beam is applied on the top surface $(z=0)$ at $t=0$ and the peak intensity occurs when $t=2 t_{p}$.

For micro spheres as shown in Fig. (1b), the above governing equations may be transformed to the equations under spherical coordinates

$((r, \theta, \varphi) ; 0 \leq \theta \leq 2 \pi, 0 \leq \varphi \leq \pi)$ as follows:

(1') Dynamic Equations of Motion [8, 4]

$$
\begin{aligned}
& \rho^{(m)} \frac{\partial^{2} u_{r}^{(m)}}{\partial t^{2}} \\
& =\frac{\partial \sigma_{r}^{(m)}}{\partial r}+\frac{1}{r} \frac{\partial \sigma_{r \varphi}^{(m)}}{\partial \varphi}+\frac{1}{r \sin \varphi} \frac{\partial \sigma_{r \theta}^{(m)}}{\partial \theta} \\
& +\frac{1}{r}\left(2 \sigma_{r}^{(m)}-\sigma_{\varphi}^{(m)}-\sigma_{\theta}^{(m)}+\sigma_{r \varphi}^{(m)} \cot \varphi\right) \\
& +2 \Lambda^{(m)} T_{e}^{(m)} \frac{\partial T_{e}^{(m)}}{\partial r}, \\
& \rho{ }^{(m)} \frac{\partial^{2} u_{\varphi}^{(m)}}{\partial t^{2}} \\
& =\frac{\partial \sigma_{r \varphi}^{(m)}}{\partial r}+\frac{1}{r} \frac{\partial \sigma_{\varphi}^{(m)}}{\partial \varphi}+\frac{1}{r \sin \varphi} \frac{\partial \sigma_{\varphi \theta}^{(m)}}{\partial \theta} \\
& +\frac{1}{r}\left[\left(\sigma_{\varphi}^{(m)}-\sigma_{\theta}^{(m)}\right) \cot \varphi+3 \sigma_{r \varphi}^{(m)}\right] \\
& +2 \Lambda^{(m)} T_{e}^{(m)} \frac{1}{r} \frac{\partial T_{e}^{(m)}}{\partial \varphi},
\end{aligned}
$$




$$
\begin{aligned}
& \rho^{(m)} \frac{\partial^{2} u_{\theta}^{(m)}}{\partial t^{2}} \\
& =\frac{\partial \sigma_{r \theta}^{(m)}}{\partial r}+\frac{1}{r} \frac{\partial \sigma_{\varphi \theta}^{(m)}}{\partial \varphi}+\frac{1}{r \sin \varphi} \frac{\partial \sigma_{\theta}^{(m)}}{\partial \theta} \\
& +\frac{1}{r}\left(2 \sigma_{\varphi \theta}^{(m)} \cot \varphi+3 \sigma_{r \theta}^{(m)}\right) \\
& +2 \Lambda^{(m)} T_{e}^{(m)} \frac{1}{r \sin \varphi} \frac{\partial T_{e}^{(m)}}{\partial \theta},
\end{aligned}
$$

where

$$
\begin{aligned}
& \sigma_{r}^{(m)}=\lambda^{(m)}\left(\varepsilon_{r}^{(m)}+\varepsilon_{\theta}^{(m)}+\varepsilon_{\varphi}^{(m)}\right)+2 \mu^{(m)} \varepsilon_{r}^{(m)} \\
& -\left(3 \lambda^{(m)}+2 \mu^{(m)}\right) \alpha_{T}^{(m)}\left(T_{l}^{(m)}-T_{0}\right), \\
& \sigma_{\theta}^{(m)}=\lambda^{(m)}\left(\varepsilon_{r}^{(m)}+\varepsilon_{\theta}^{(m)}+\varepsilon_{\varphi}^{(m)}\right)+2 \mu^{(m)} \varepsilon_{\theta}^{(m)} \\
& -\left(3 \lambda^{(m)}+2 \mu^{(m)}\right) \alpha_{T}^{(m)}\left(T_{l}^{(m)}-T_{0}\right), \\
& \sigma_{\varphi}^{(m)}=\lambda^{(m)}\left(\varepsilon_{r}^{(m)}+\varepsilon_{\theta}^{(m)}+\varepsilon_{\varphi}^{(m)}\right)+2 \mu^{(m)} \varepsilon_{\varphi}^{(m)} \\
& -\left(3 \lambda^{(m)}+2 \mu^{(m)}\right) \alpha_{T}^{(m)}\left(T_{l}^{(m)}-T_{0}\right), \\
& \sigma_{r \theta}^{(m)}=2 \mu^{(m)} \varepsilon_{r \theta}^{(m)}, \sigma_{r \varphi}^{(m)}=2 \mu^{(m)} \varepsilon_{r \varphi}^{(m)}, \\
& \sigma_{\varphi \theta}^{(m)}=2 \mu^{(m)} \varepsilon_{\varphi \theta}^{(m)}, \\
& \varepsilon_{r}^{(m)}=\frac{\partial u_{r}^{(m)}}{\partial r}, \varepsilon_{\varphi}^{(m)}=\frac{1}{r}\left(u_{r}^{(m)}+\frac{\partial u_{\varphi}^{(m)}}{\partial \varphi}\right), \\
& \varepsilon_{\theta}^{(m)}=\frac{1}{r \sin \varphi}\left(\frac{\partial u_{\theta}^{(m)}}{\partial \theta}+\sin \varphi u_{r}^{(m)}+\cos \varphi u_{\varphi}^{(m)}\right), \\
& \varepsilon_{r \varphi}^{(m)}=\frac{1}{2}\left(\frac{1}{r} \frac{\partial u_{r}^{(m)}}{\partial \varphi}+\frac{\partial u_{\varphi}^{(m)}}{\partial r}-\frac{u_{\varphi}^{(m)}}{r}\right), \\
& \varepsilon_{\varphi \theta}^{(m)}=\frac{1}{2 r}\left(\frac{1}{\sin \varphi} \frac{\partial u_{\varphi}^{(m)}}{\partial \theta}+\frac{\partial u_{\theta}^{(m)}}{\partial \varphi}-u_{\theta}^{(m)} \cot \varphi\right), \\
& \varepsilon_{r \theta}^{(m)}=\frac{1}{2}\left(\frac{1}{r \sin \varphi} \frac{\partial u_{r}^{(m)}}{\partial \theta}+\frac{\partial u_{\theta}^{(m)}}{\partial r}-\frac{u_{\theta}^{(m)}}{r}\right) .
\end{aligned}
$$

Here, $m=1,2$ denote the outside layer and the inside layer, respectively.

(2') Energy Equations [8, 15]

$$
\begin{aligned}
& C_{e}^{(m)}\left(T_{e}\right) \frac{\partial T_{e}^{(m)}}{\partial t} \\
& =\frac{1}{r^{2}} \frac{\partial}{\partial r}\left(k_{e}^{(m)}\left(T_{e}, T_{l}\right) r^{2} \frac{\partial T_{e}^{(m)}}{\partial r}\right) \\
& +\frac{1}{r^{2} \sin ^{2} \varphi} \frac{\partial}{\partial \theta}\left(k_{e}^{(m)}\left(T_{e}, T_{l}\right) \frac{\partial T_{e}^{(m)}}{\partial \theta}\right) \\
& +\frac{1}{r^{2} \sin \varphi} \frac{\partial}{\partial \varphi}\left(k_{e}^{(m)}\left(T_{e}, T_{l}\right) \sin \varphi \frac{\partial T_{e}^{(m)}}{\partial \varphi}\right) \\
& -G^{(m)}\left(T_{e}^{(m)}-T_{l}^{(m)}\right)+Q,
\end{aligned}
$$

$$
\begin{aligned}
C_{l}^{(m)} \frac{\partial T_{l}^{(m)}}{\partial t} & =G^{(m)}\left(T_{e}^{(m)}-T_{l}^{(m)}\right) \\
& -\left(3 \lambda^{(m)}+2 \mu^{(m)}\right) \alpha_{T}^{(m)} T_{0} \\
& \times \frac{\partial}{\partial t}\left(\varepsilon_{r}^{(m)}+\varepsilon_{\theta}^{(m)}+\varepsilon_{\varphi}^{(m)}\right),
\end{aligned}
$$

where the heat source term, which is obtained based on that in Eq. (7) under spherical coordinates, is given by

$Q(r, \theta, \varphi, t)$

$=0.94 J \frac{1-R}{t_{p} \zeta} \exp \left(-\frac{L-r}{\zeta}-2.77\left(\frac{t-2 t_{p}}{t_{p}}\right)^{2}\right) \cos \varphi$.

Here, $L$ is the radius of the micro-sphere and $\zeta$ is the optical penetration depth.

\section{FINITE DIFFERENCE METHOD}

Following the approach in [2-4, 9], we introduce three velocity components $v_{1}, v_{2}$ and $v_{3}$ into the model and rewrite the dynamic equations of motion, Eqs. (1)-(4), as follows:

$$
\begin{aligned}
& v_{1}^{(m)}=\frac{\partial u^{(m)}}{\partial t}, v_{2}^{(m)}=\frac{\partial v^{(m)}}{\partial t}, v_{3}^{(m)}=\frac{\partial w^{(m)}}{\partial t}, \\
& \rho^{(m)} \frac{\partial v_{1}^{(m)}}{\partial t}=\frac{\partial \sigma_{x}^{(m)}}{\partial x}+\frac{\partial \sigma_{x y}^{(m)}}{\partial y}+\frac{\partial \sigma_{x z}^{(m)}}{\partial z} \\
& +2 \Lambda^{(m)} T_{e}^{(m)} \frac{\partial T_{e}^{(m)}}{\partial x}, \\
& \rho^{(m)} \frac{\partial v_{2}^{(m)}}{\partial t}=\frac{\partial \sigma_{x y}^{(m)}}{\partial x}+\frac{\partial \sigma_{y}^{(m)}}{\partial y}+\frac{\partial \sigma_{y z}^{(m)}}{\partial z} \\
& +2 \Lambda^{(m)} T_{e}^{(m)} \frac{\partial T_{e}^{(m)}}{\partial y}, \\
& \rho^{(m)} \frac{\partial v_{3}^{(m)}}{\partial t}=\frac{\partial \sigma_{x z}^{(m)}}{\partial x}+\frac{\partial \sigma_{y z}^{(m)}}{\partial y}+\frac{\partial \sigma_{z}^{(m)}}{\partial z} \\
& +2 \Lambda^{(m)} T_{e}^{(m)} \frac{\partial T_{e}^{(m)}}{\partial z}, \\
& \frac{\partial \varepsilon_{x}^{(m)}}{\partial t}=\frac{\partial v_{1}^{(m)}}{\partial x}, \frac{\partial \varepsilon_{y}^{(m)}}{\partial t}=\frac{\partial v_{2}^{(m)}}{\partial y}, \\
& \frac{\partial \varepsilon_{z}^{(m)}}{\partial t}=\frac{\partial v_{3}^{(m)}}{\partial z} \\
& \frac{\partial \gamma_{x y}^{(m)}}{\partial t}=\frac{\partial v_{1}^{(m)}}{\partial y}+\frac{\partial v_{2}^{(m)}}{\partial x}, \\
& \frac{\partial \gamma_{x z}^{(m)}}{\partial t}=\frac{\partial v_{1}^{(m)}}{\partial z}+\frac{\partial v_{3}^{(m)}}{\partial x}, \\
& \frac{\partial \gamma_{y z}^{(m)}}{\partial t}=\frac{\partial v_{2}^{(m)}}{\partial z}+\frac{\partial v_{3}^{(m)}}{\partial y} \text {. }
\end{aligned}
$$

To develop a finite difference scheme, we first construct a staggered grid as shown in Fig. (2), where $v_{1}^{(m)}$ is placed 

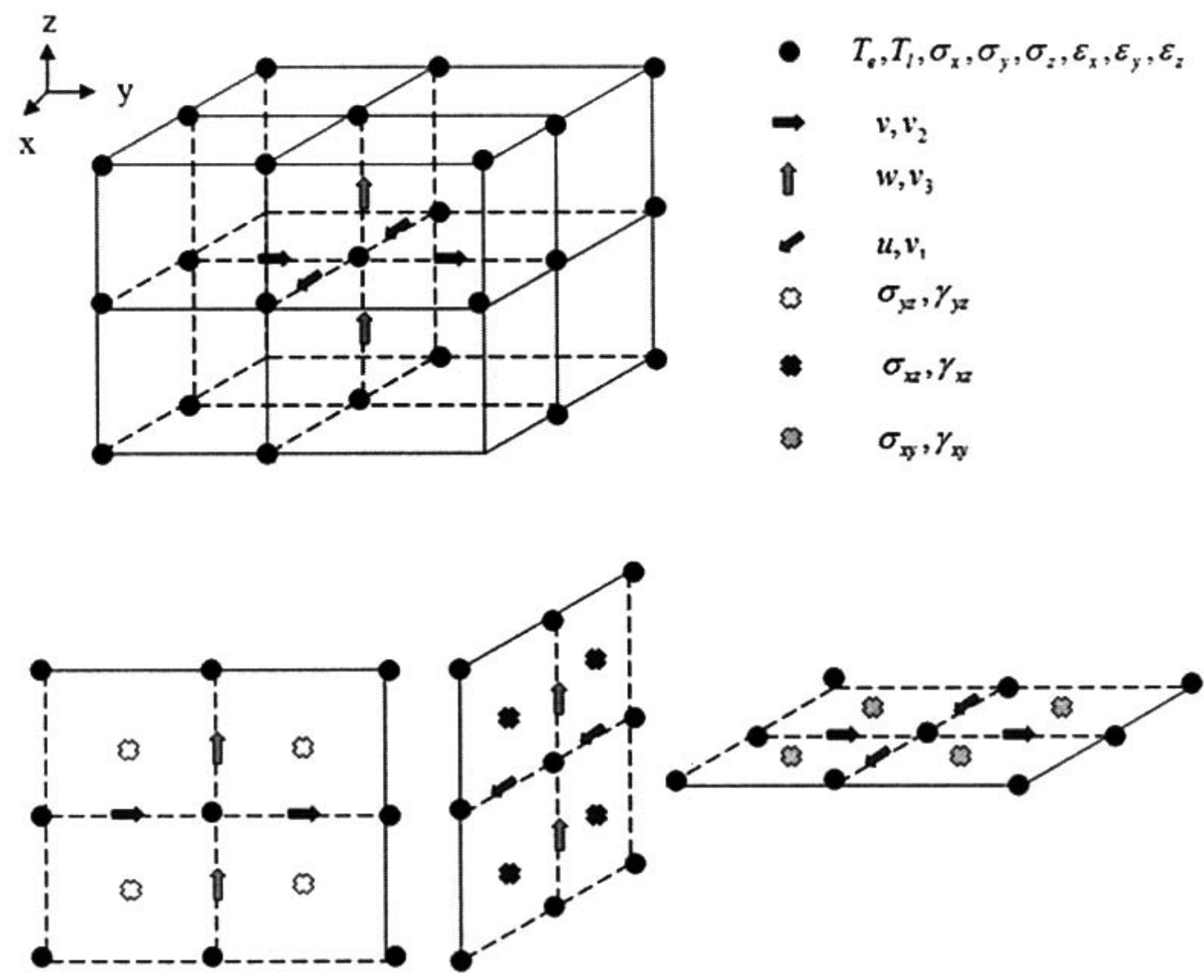

Fig. (2A). 3D staggered mesh for a thin film and locations of variables.

at $\left(x_{i+\frac{1}{2}}, y_{j}, z_{k}\right), v_{2}^{(m)}$ is placed at $\left(x_{i}, y_{j+\frac{1}{2}}, z_{k}\right), v_{3}^{(m)}$ is placed at $\left(x_{i}, y_{j}, z_{k+\frac{1}{2}}\right), \gamma_{x y}^{(m)}$ and $\sigma_{x y}^{(m)}$ are placed at $\left(x_{i+\frac{1}{2}}, y_{j+\frac{1}{2}}, z_{k}\right), \gamma_{x z}^{(m)}$ and $\sigma_{x z}^{(m)}$ are placed at $\left(x_{i+\frac{1}{2}}, y_{j}, z_{k+\frac{1}{2}}\right), \quad \gamma_{y z}^{(m)}$ and $\sigma_{y z}^{(m)}$ are placed at $\left(x_{i}, y_{j+\frac{1}{2}}, z_{k+\frac{1}{2}}\right), \quad$ while $\quad \varepsilon_{x}^{(m)}, \quad \varepsilon_{y}^{(m)}, \quad \varepsilon_{z}^{(m)}$, $\sigma_{x}^{(m)}, \sigma_{y}^{(m)}, \sigma_{z}^{(m)}, T_{e}^{(m)}$ and $T_{l}^{(m)}$ are at $\left(x_{i}, y_{j}, z_{k}\right)$. Here, $i, j$ and $k$ are indices with $1 \leq i \leq N_{x}+1$, $1 \leq j \leq N_{y}+1$, and $1 \leq k \leq N_{z}+1$, such that $N_{x} \Delta x=L_{x}$, $N_{y} \Delta y=L_{y}$ and $N_{z} \Delta z=L_{z}$, where $\Delta x, \Delta y$ and $\Delta z$ are spatial step sizes. We denote $\left(v_{1}^{(m)}\right)_{i+\frac{1}{2}, j, k}^{n},\left(v_{2}^{(m)}\right)_{i, j+\frac{1}{2}, k}^{n}$ and $\left(v_{3}^{(m)}\right)_{i, j, k+\frac{1}{2}}^{n}$ as numerical approximations of $v_{1}^{(m)}\left(\left(i+\frac{1}{2}\right) \Delta x, j \Delta y, k \Delta z, n \Delta t\right)$ $v_{2}^{(m)}\left(i \Delta x,\left(j+\frac{1}{2}\right) \Delta y, k \Delta z, n \Delta t\right)$ and $v_{3}^{(m)}\left(i \Delta x, j \Delta y,\left(k+\frac{1}{2}\right) \Delta z, n \Delta t\right)$, respectively, where $\Delta t$ is the time increment. Similar notations are used for other variables. Furthermore, we introduce the finite difference operators, $\Delta_{-t}$ and $\delta_{x}$, as follows:

$\Delta_{-t} u_{i, j, k}^{n}=u_{i, j, k}^{n}-u_{i, j, k}^{n-1}$,
$\delta_{x} u_{i, j, k}^{n}=u_{i+\frac{1}{2}, j, k}^{n}-u_{i-\frac{1}{2}, j, k}^{n}$.

$\delta_{y}$ and $\delta_{z}$ are defined similar to $\delta_{x}$.

To avoid non-physical oscillations in the solution, we further follow the approach in [2-4, 9] and employ a fourthorder compact finite difference scheme for obtaining stress derivatives, $\frac{\partial \sigma_{x}}{\partial x}, \frac{\partial \sigma_{x y}}{\partial y}, \frac{\partial \sigma_{x z}}{\partial z}$ the etc. in Eqs. (19)-(21).

For example, $\frac{\partial \sigma_{x}}{\partial x}$ can be obtained by solving the following tridiagonal linear system (indices $j$ and $k$ are omitted). 


$$
\begin{aligned}
& \frac{1}{24} \frac{\partial\left(\sigma_{x}^{(m)}\right)_{i-1}}{\partial x}+\frac{11}{12} \frac{\partial\left(\sigma_{x}^{(m)}\right)_{i}}{\partial x}+\frac{1}{24} \frac{\partial\left(\sigma_{x}^{(m)}\right)_{i+1}}{\partial x} \\
& =\frac{\left(\sigma_{x}^{(m)}\right)_{i+\frac{1}{2}}-\left(\sigma_{x}^{(m)}\right)_{i-\frac{1}{2}}}{\Delta x}, 2+\frac{1}{2} \leq i \leq N_{x}-\frac{1}{2},
\end{aligned}
$$

where

$$
\begin{aligned}
& \frac{\partial\left(\sigma_{x}^{(m)}\right) \frac{3}{2}}{\partial x}=\frac{\left(\sigma_{x}^{(m)}\right)_{2}-\left(\sigma_{x}^{(m)}\right)_{1}}{\Delta x}, \\
& \frac{\partial\left(\sigma_{x}^{(m)}\right)_{N_{x}+\frac{1}{2}}}{\partial x}=\frac{\left(\sigma_{x}^{(m)}\right)_{N_{x}+1}-\left(\sigma_{x}^{(m)}\right)_{N_{x}}}{\Delta x} .
\end{aligned}
$$

As such, the implicit finite difference schemes for solving Eqs. (18)-(21) coupled with Eqs. (4a)-(4f) can be written as follows:

$$
\begin{aligned}
& \rho^{(m)} \frac{1}{\Delta t} \Delta_{-t}\left(v_{1}^{(m)}\right)_{i+\frac{1}{2}, j, k}^{n+1} \\
& =\frac{\partial\left(\sigma_{x}^{(m)}\right)_{i+\frac{1}{2}, j, k}^{n+1}}{\partial x}+\frac{\partial\left(\sigma_{x y}^{(m)}\right)_{i+\frac{1}{2}, j, k}^{n+1}}{\partial y}+\frac{\partial\left(\sigma_{x z}^{(m)}\right)_{i+\frac{1}{2}, j, k}^{n+1}}{\partial z} \\
& +\Lambda^{(m)} \frac{1}{\Delta x} \delta_{x}\left(\left(T_{e}^{(m)}\right)_{i+\frac{1}{2}, j, k}^{n+1}\right)^{2}, \\
& \rho^{(m)} \frac{1}{\Delta t} \Delta_{-t}\left(v_{2}^{(m)}\right)_{i, j+\frac{1}{2}, k}^{n+1} \\
& =\frac{\partial\left(\sigma_{y}^{(m)}\right)_{i, j+\frac{1}{2}, k}^{n+1}}{\partial y}+\frac{\partial\left(\sigma_{x y}^{(m)}\right)_{i, j+\frac{1}{2}, k}^{n+1}}{\partial x}+\frac{\partial\left(\sigma_{y z}^{(m)}\right)_{i, j+\frac{1}{2}, k}^{n+1}}{\partial z} \\
& +\Lambda^{(m)} \frac{1}{\Delta y} \delta_{y}\left(\left(T_{e}^{(m)}\right)_{i, j+\frac{1}{2}, k}^{n+1}\right)^{2}, \\
& \rho^{(m)} \frac{1}{\Delta t} \Delta_{-t}\left(v_{3}^{(m)}\right)_{i, j, k+\frac{1}{2}}^{n+1} \\
& =\frac{\partial\left(\sigma_{z}^{(m)}\right)_{i, j, k+\frac{1}{2}}^{n+1}}{\partial z}+\frac{\partial\left(\sigma_{x z}^{(m)}\right)_{i, j, k+\frac{1}{2}}^{n+1}}{\partial x}+\frac{\partial\left(\sigma_{y z}^{(m)}\right)_{i, j, k+\frac{1}{2}}^{n+1}}{\partial y} \\
& +\Lambda^{(m)} \frac{1}{\Delta z} \delta_{z}\left(\left(T_{e}^{(m)}\right)_{i, j, k+\frac{1}{2}}^{n+1}\right)^{2} . \\
& \frac{1}{\Delta t} \Delta_{-t}\left(\varepsilon_{x}^{(m)}\right)_{i, j, k}^{n+1}=\frac{1}{\Delta x} \delta_{x}\left(v_{1}^{(m)}\right)_{i, j, k}^{n+1} \text {, } \\
& \frac{1}{\Delta t} \Delta_{-t}\left(\varepsilon_{y}^{(m)}\right)_{i, j, k}^{n+1}=\frac{1}{\Delta y} \delta_{y}\left(v_{2}^{(m)}\right)_{i, j, k}^{n+1}, \\
& \frac{1}{\Delta t} \Delta_{-t}\left(\varepsilon_{z}^{(m)}\right)_{i, j, k}^{n+1}=\frac{1}{\Delta z} \delta_{z}\left(v_{3}^{(m)}\right)_{i, j, k}^{n+1},
\end{aligned}
$$

$$
\begin{aligned}
\frac{1}{\Delta t} \Delta_{-t}\left(\gamma_{x y}^{(m)}\right)_{i+\frac{1}{2}, j+\frac{1}{2}, k}^{n+1} & =\frac{1}{\Delta y} \delta_{y}\left(v_{1}^{(m)}\right)_{i+\frac{1}{2}, j+\frac{1}{2}, k}^{n+1} \\
& +\frac{1}{\Delta x} \delta_{x}\left(v_{2}^{(m)}\right)^{n+1}{ }_{i+\frac{1}{2}, j+\frac{1}{2}, k}, \\
\frac{1}{\Delta t} \Delta_{-t}\left(\gamma_{x z}^{(m)}\right)_{i+\frac{1}{2}, j, k+\frac{1}{2}}^{n+1} & =\frac{1}{\Delta z} \delta_{z}\left(v_{1}^{(m)}\right)_{i+\frac{1}{2}, j, k+\frac{1}{2}}^{n+1} \\
& +\frac{1}{\Delta x} \delta_{x}\left(v_{3}^{(m)}\right)_{i+\frac{1}{2}, j, k+\frac{1}{2}}^{n+1},
\end{aligned}
$$$$
\frac{1}{\Delta t} \Delta_{-t}\left(\gamma_{y z}^{(m)}\right)_{i, j+\frac{1}{2}, k+\frac{1}{2}}^{n+1}=\frac{1}{\Delta z} \delta_{z}\left(v_{2}^{(m)}\right)_{i, j+\frac{1}{2}, k+\frac{1}{2}}^{n+1}
$$$$
+\frac{1}{\Delta y} \delta_{y}\left(v_{3}^{(m)}\right)_{i, j+\frac{1}{2}, k+\frac{1}{2}}^{n+1},
$$

$$
\begin{aligned}
\left(\sigma_{x}^{(m)}\right)_{i, j . k}^{n+1} & =\lambda^{(m)}\left[\left(\varepsilon_{x}^{(m)}\right)_{i, j . k}^{n+1}+\left(\varepsilon_{y}^{(m)}\right)_{i, j . k}^{n+1}\right. \\
& \left.+\left(\varepsilon_{z}^{(m)}\right)_{i, j . k}^{n+1}\right]+2 \mu^{(m)}\left(\varepsilon_{x}^{(m)}\right)_{i, j . k}^{n+1} \\
& -\left(3 \lambda^{(m)}+2 \mu^{(m)}\right) \alpha_{T}^{(m)} \\
& \times\left[\left(T_{l}^{(m)}\right)_{i, j . k}^{n+1}-T_{0}\right],
\end{aligned}
$$$$
\left(\sigma_{y}^{(m)}\right)_{i, j . k}^{n+1}=\lambda^{(m)}\left[\left(\varepsilon_{x}^{(m)}\right)_{i, j . k}^{n+1}+\left(\varepsilon_{y}^{(m)}\right)_{i, j . k}^{n+1}\right.
$$$$
\left.+\left(\varepsilon_{z}^{(m)}\right)_{i, j . k}^{n+1}\right]+2 \mu^{(m)}\left(\varepsilon_{y}^{(m)}\right)_{i, j . k}^{n+1}
$$$$
-\left(3 \lambda^{(m)}+2 \mu^{(m)}\right) \alpha_{T}^{(m)}\left[\left(T_{l}^{(m)}\right)_{i, j . k}^{n+1}-T_{0}\right],
$$$$
\left(\sigma_{z}^{(m)}\right)_{i, j . k}^{n+1}=\lambda^{(m)}\left[\left(\varepsilon_{x}^{(m)}\right)_{i, j . k}^{n+1}+\left(\varepsilon_{y}^{(m)}\right)_{i, j . k}^{n+1}\right.
$$$$
\left.+\left(\varepsilon_{z}^{(m)}\right)_{i, j . k}^{n+1}\right]+2 \mu^{(m)}\left(\varepsilon_{z}^{(m)}\right)_{i, j . k}^{n+1}
$$$$
-\left(3 \lambda^{(m)}+2 \mu^{(m)}\right) \alpha_{T}^{(m)}\left[\left(T_{l}^{(m)}\right)_{i, j . k}^{n+1}-T_{0}\right],
$$$$
\left(\sigma_{x y}^{(m)}\right)_{i+\frac{1}{2}, j+\frac{1}{2}, k}^{n+1}=\mu^{(m)}\left(\gamma_{x y}^{(m)}\right)_{i+\frac{1}{2}, j+\frac{1}{2}, k}^{n+1},
$$$$
\left(\sigma_{x z}^{(m)}\right)_{i+\frac{1}{2}, j, k+\frac{1}{2}}^{n+1}=\mu^{(m)}\left(\gamma_{x z}^{(m)}\right)_{i+\frac{1}{2}, j, k+\frac{1}{2}}^{n+1},
$$$$
\left(\sigma_{y z}^{(m)}\right)_{i, j+\frac{1}{2}, k+\frac{1}{2}}^{n+1}=\mu^{(m)}\left(\gamma_{y z}^{(m)}\right)_{i, j+\frac{1}{2}, k+\frac{1}{2}}^{n+1},
$$$$
\frac{1}{\Delta t} \Delta_{-t}\left(u^{(m)}\right)_{i+\frac{1}{2}, j, k}^{n+1}=\left(v_{1}^{(m)}\right)_{i+\frac{1}{2}, j, k}^{n+1},
$$$$
\frac{1}{\Delta t} \Delta_{-t}\left(v^{(m)}\right)_{i, j+\frac{1}{2}, k}^{n+1}=\left(v_{2}^{(m)}\right)_{i, j+\frac{1}{2}, k}^{n+1},
$$$$
\frac{1}{\Delta t} \Delta_{-t}\left(w^{(m)}\right)_{i, j, k+\frac{1}{2}}^{n+1}=\left(v_{3}^{(m)}\right)_{i, j, k+\frac{1}{2}}^{n+1} .
$$

On the other hand, the energy equations, Eqs. (15)-(16), are solved using the Crank-Nicholson finite difference method [16]: 


$$
\begin{aligned}
& C_{e 0}^{(m)} \frac{\left(T_{e}^{(m)}\right)_{i, j, k}^{n+1}+\left(T_{e}^{(m)}\right)_{i, j, k}^{n}}{2 T_{0}} \cdot \frac{1}{\Delta t} \Delta_{-t}\left(T_{e}^{(m)}\right)_{i, j, k}^{n+1} \\
& =\frac{1}{2 \Delta x^{2}}\left(\left(k_{e}^{(m)}\right)^{n+1}{ }_{i+\frac{1}{2}, j, k}^{n+1} \delta_{x}\left(T_{e}^{(m)}\right)^{n+1}{ }_{i+\frac{1}{2}, j, k}\right. \\
& \left.-\left(k_{e}^{(m)}\right)_{i-\frac{1}{2}, j, k}^{n+1} \delta_{x}\left(T_{e}^{(m)}\right)_{i-\frac{1}{2}, j, k}^{n+1}\right) \\
& +\frac{1}{2 \Delta x^{2}}\left(\left(k_{e}^{(m)}\right)_{i+\frac{1}{2}, j, k}^{n} \delta_{x}\left(T_{e}^{(m)}\right)_{i+\frac{1}{2}, j, k}^{n}\right. \\
& \left.-\left(k_{e}^{(m)}\right)_{i-\frac{1}{2}, j, k}^{n} \delta_{x}\left(T_{e}^{(m)}\right)_{i-\frac{1}{2}, j, k}^{n}\right) \\
& +\frac{1}{2 \Delta y^{2}}\left(\left(k_{e}^{(m)}\right)_{i, j+\frac{1}{2}, k}^{n+1} \delta_{y}\left(T_{e}^{(m)}\right)_{i, j+\frac{1}{2}, k}^{n+1}\right. \\
& \left.-\left(k_{e}^{(m)}\right)_{i, j-\frac{1}{2}, k}^{n+1} \delta_{y}\left(T_{e}^{(m)}\right)_{i, j-\frac{1}{2}, k}^{n+1}\right) \\
& +\frac{1}{2 \Delta y^{2}}\left(\left(k_{e}^{(m)}\right)_{i, j+\frac{1}{2}, k}^{n} \delta_{y}\left(T_{e}^{(m)}\right)_{i, j+\frac{1}{2}, k}^{n}\right. \\
& \left.-\left(k_{e}^{(m)}\right)_{i, j-\frac{1}{2}, k}^{n} \delta_{y}\left(T_{e}^{(m)}\right)_{i, j-\frac{1}{2}, k}^{n}\right) \\
& +\frac{1}{2 \Delta z^{2}}\left(\left(k_{e}^{(m)}\right)_{i, j, k+\frac{1}{2}}^{n+1} \delta_{z}\left(T_{e}^{(m)}\right)_{i, j, k+\frac{1}{2}}^{n+1}\right. \\
& \left.-\left(k_{e}^{(m)}\right)_{i, j, k-\frac{1}{2}}^{n+1} \delta_{z}\left(T_{e}^{(m)}\right)_{i, j, k-\frac{1}{2}}^{n+1}\right) \\
& +\frac{1}{2 \Delta z^{2}}\left(\left(k_{e}^{(m)}\right)_{i, j, k+\frac{1}{2}}^{n} \delta_{z}\left(T_{e}^{(m)}\right)_{i, j, k+\frac{1}{2}}^{n}\right. \\
& \left.-\left(k_{e}^{(m)}\right)_{i, j, k-\frac{1}{2}}^{n} \delta_{z}\left(T_{e}^{(m)}\right)_{i, j, k-\frac{1}{2}}^{n}\right) \\
& -G^{(m)}\left(\frac{\left(T_{e}^{(m)}\right)_{i, j, k}^{n+1}+\left(T_{e}^{(m)}\right)_{i, j, k}^{n}}{2}\right. \\
& \left.-\frac{\left(T_{l}^{(m)}\right)_{i, j, k}^{n+1}+\left(T_{l}^{(m)}\right)_{i, j, k}^{n}}{2}\right)+Q_{i, j, k}^{n+\frac{1}{2}}, \\
& C_{l}^{(m)} \frac{1}{\Delta t} \Delta_{-t}\left(T_{l}^{(m)}\right)_{i, j, k}^{n+1} \\
& =G^{(m)}\left(\frac{\left(T_{e}^{(m)}\right)_{i, j, k}^{n+1}+\left(T_{e}^{(m)}\right)_{i, j, k}^{n}}{2}\right. \\
& \left.-\frac{\left(T_{l}^{(m)}\right)_{i, j, k}^{n+1}+\left(T_{l}^{(m)}\right)_{i, j, k}^{n}}{2}\right) \\
& -\left(3 \lambda^{(m)}+2 \mu^{(m)}\right) \frac{\alpha_{T}^{(m)}}{\Delta t} T_{0}\left(\Delta_{-t}\left(\varepsilon_{x}^{(m)}\right)_{i, j, k}^{n+1}\right. \\
& \left.+\Delta_{-t}\left(\varepsilon_{y}^{(m)}\right)_{i, j, k}^{n+1}+\Delta_{-t}\left(\varepsilon_{z}^{(m)}\right)_{i, j, k}^{n+1}\right),
\end{aligned}
$$

The interfacial conditions for the velocity components $v_{1}^{(m)}, v_{2}^{(m)}$, and $v_{3}^{(m)}$ are obtained based on Eq. (9a)

$$
\begin{aligned}
& \left(v_{1}^{(1)}\right)_{i+\frac{1}{2}, j, N+1}^{n+1}=\left(v_{1}^{(2)}\right)_{i+\frac{1}{2}, j, 1}^{n+1}, \\
& \left(v_{2}^{(1)}\right)_{i, j+\frac{1}{2}, N+1}^{n+1}=\left(v_{2}^{(2)}\right)_{i, j+\frac{1}{2}, 1}^{n+1}, \\
& \left(v_{3}^{(1)}\right)_{i, j, N+\frac{1}{2}}^{n+1}=\left(v_{3}^{(2)}\right)_{i, j, \frac{3}{2}}^{n+1},
\end{aligned}
$$

and from Eqs. (9b) and (9c)

$$
\begin{aligned}
& \left(\sigma_{z}^{(1)}\right)_{i, j, N+1}^{n+1}=\left(\sigma_{z}^{(2)}\right)_{i, j, 1}^{n+1}, \\
& \left(\sigma_{x z}^{(1)}\right)_{i+\frac{1}{2}, j, N+\frac{1}{2}}^{n+1}=\left(\sigma_{x z}^{(2)}\right)_{i+\frac{1}{2}, j, \frac{3}{2}}^{n+1}, \\
& \left(\sigma_{y z}^{(1)}\right)_{i, j+\frac{1}{2}, N+\frac{1}{2}}^{n+1}=\left(\sigma_{y z}^{(2)}\right)_{i, j+\frac{1}{2}, \frac{3}{2}}^{n+1}, \\
& \left(k_{e}^{(1)}\right)_{i, j, N+\frac{1}{2}}^{n+1} \frac{\left(T_{e}^{(1)}\right)_{i, j, N+1}^{n+1}-\left(T_{e}^{(1)}\right)_{i, j, N}^{n+1}}{\Delta z} \\
& =\left(k_{e}^{(2)}\right)_{i, j, \frac{3}{2}}^{n+1} \frac{\left(T_{e}^{(2)}\right)_{i, j, 2}^{n+1}-\left(T_{e}^{(2)}\right)_{i, j, 1}^{n+1}}{\Delta z}, \\
& \left(T_{e}^{(1)}\right)_{i, j, N+1}^{n+1}=\left(T_{e}^{(2)}\right)_{i, j, 1}^{n+1} .
\end{aligned}
$$

It should be pointed out that Eqs. (25)-(27) are nonlinear since the terms $\delta_{x}\left(\left(T_{e}^{(m)}\right)_{i+\frac{1}{2}, j, k}^{n+1}\right)^{2}, \quad \delta_{y}\left(\left(T_{e}^{(m)}\right)_{i, j+\frac{1}{2}, k}^{n+1}\right)^{2}$ and $\delta_{z}\left(\left(T_{e}^{(m)}\right)_{i, j, k+\frac{1}{2}}^{n+1}\right)^{2}$ are nonlinear. Also, it can be seen that Eq. (33) is nonlinear. Therefore, the above scheme must be solved iteratively. An iterative method for solving the above scheme at time level $n+1$ is developed as follows:

Step 1 . Set the initial values for $\left(\varepsilon_{x}^{(m)}\right)^{n+1},\left(\varepsilon_{y}^{(m)}\right)^{n+1}$, $\left(\varepsilon_{z}^{(m)}\right)^{n+1},\left(\gamma_{x y}^{(m)}\right)^{n+1},\left(\gamma_{x z}^{(m)}\right)^{n+1}$ and $\left(\gamma_{y z}^{(m)}\right)^{n+1}$, solve iteratively Eqs. (33) and (34) coupled with the interfacial conditions, Eqs. (35e)-(35f), for $\left(T_{e}^{(m)}\right)^{n+1}$ and $\left(T_{l}^{(m)}\right)^{n+1}$.

Step 2. Solve for $\left(\sigma_{x}^{(m)}\right)^{n+1},\left(\sigma_{y}^{(m)}\right)^{n+1},\left(\sigma_{z}^{(m)}\right)^{n+1}$, $\left(\sigma_{x y}^{(m)}\right)^{n+1},\left(\sigma_{x z}^{(m)}\right)^{n+1}$ and $\left(\sigma_{y z}^{(m)}\right)^{n+1}$ using Eqs. (30)-(31).

Step 3. Solve for the derivatives of $\left(\sigma_{x}^{(m)}\right)^{n+1}$, $\left(\sigma_{y}^{(m)}\right)^{n+1}, \quad\left(\sigma_{z}^{(m)}\right)^{n+1}, \quad\left(\sigma_{x y}^{(m)}\right)^{n+1}, \quad\left(\sigma_{x z}^{(m)}\right)^{n+1} \quad$ and $\left(\sigma_{y z}^{(m)}\right)^{n+1}$ using Eqs. (23)-(24) or similar equations.

Step 4. Solve for $\left(v_{1}^{(m)}\right)^{n+1},\left(v_{2}^{(m)}\right)^{n+1}$ and $\left(v_{3}^{(m)}\right)^{n+1}$ using Eqs. (25)-(27).

Step 5. Update $\left(\varepsilon_{x}^{(m)}\right)^{n+1}, \quad\left(\varepsilon_{y}^{(m)}\right)^{n+1}, \quad\left(\varepsilon_{z}^{(m)}\right)^{n+1}$, $\left(\gamma_{x y}^{(m)}\right)^{n+1},\left(\gamma_{x z}^{(m)}\right)^{n+1}$ and $\left(\gamma_{y z}^{(m)}\right)^{n+1}$, using Eqs. (28)-(29).

Given the required accuracy $\xi_{1}$ (for temperature) and $\xi_{2}$ (for strain), repeat the above steps until a convergent solution is obtained based on the following criteria. 
Table 1. Thermophysical Properties

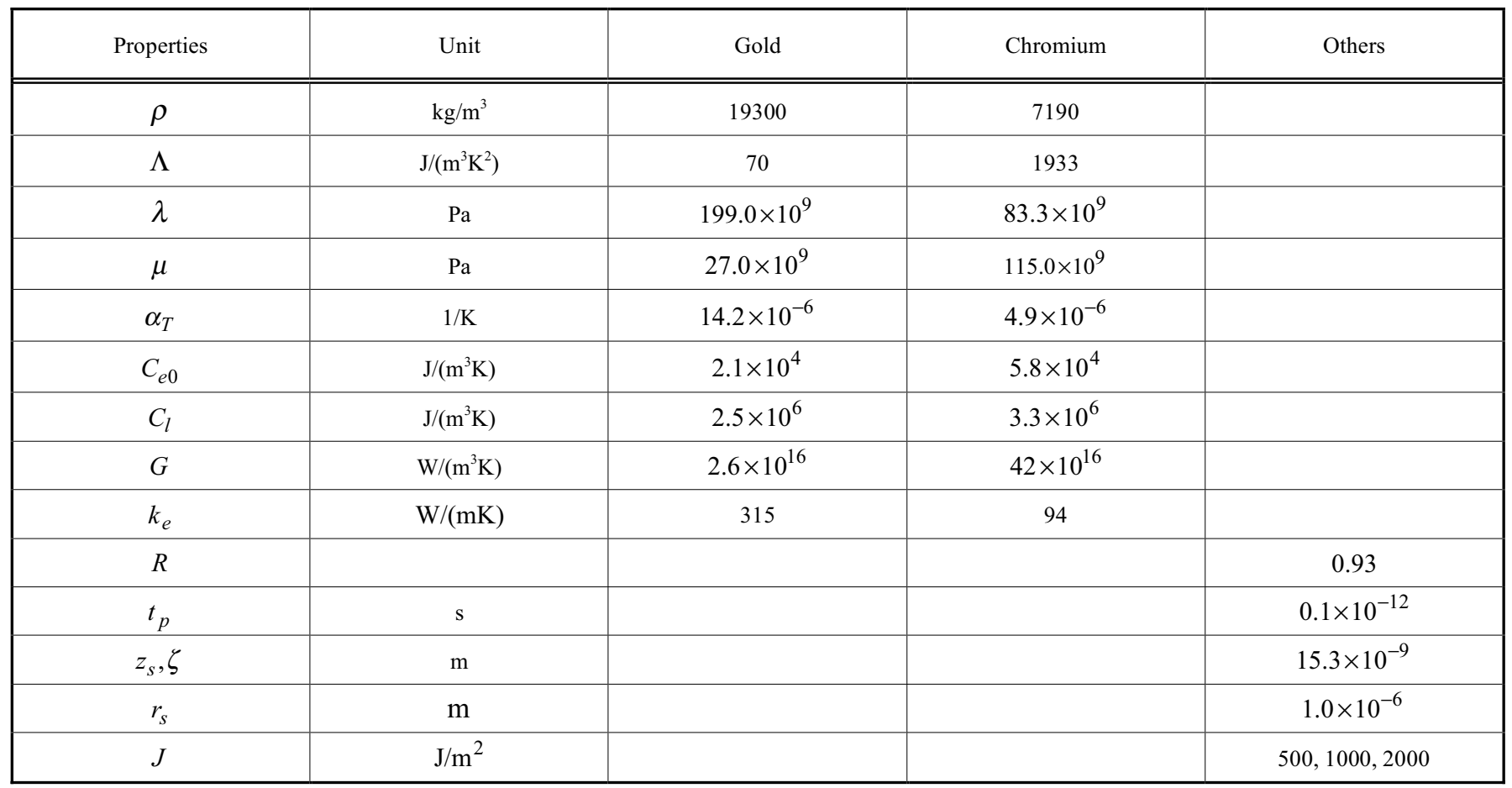

$\left|\left(T_{e}^{(m)}\right)_{i, j, k}^{n+1(\text { new })}-\left(T_{e}^{(m)}\right)_{i, j, k}^{n+1(\text { old })}\right| \leq \xi_{1}$,

$\left|\left(\varepsilon_{x}^{(m)}\right)_{i, j, k}^{n+1(\text { new })}-\left(\varepsilon_{x}^{(m)}\right)_{i, j, k}^{n+1(\text { old })}\right| \leq \xi_{2}$,

$\left|\left(\varepsilon_{y}^{(m)}\right)_{i, j, k}^{n+1(\text { new })}-\left(\varepsilon_{y}^{(m)}\right)_{i, j, k}^{n+1(\text { old })}\right| \leq \xi_{2}$,

$\left|\left(\varepsilon_{z}^{(m)}\right)_{i, j, k}^{n+1(\text { new })}-\left(\varepsilon_{z}^{(m)}\right)_{i, j, k}^{n+1(\text { old })}\right| \leq \xi_{2}$,

$\left|\left(\gamma_{x y}^{(m)}\right)_{i, j, k}^{n+1(\text { new })}-\left(\gamma_{x y}^{(m)}\right)_{i, j, k}^{n+1(\text { old })}\right| \leq \xi_{2}$,

$\left|\left(\gamma_{x z}^{(m)}\right)_{i, j, k}^{n+1(\text { new })}-\left(\gamma_{x z}^{(m)}\right)_{i, j, k}^{n+1(\text { old })}\right| \leq \xi_{2}$,

$\left|\left(\gamma_{y z}^{(m)}\right)_{i, j, k}^{n+1(\text { new })}-\left(\gamma_{y z .}^{(m)}\right)_{i, j, k}^{n+1(\text { old })}\right| \leq \xi_{2}$.

Using a similar argument, a numerical method can be obtained for studying thermal deformation in a doublelayered micro sphere induced by an ultrashort-pulsed laser.

\section{NUMERICAL EXAMPLES}

To test the applicability of the developed numerical scheme, we investigated the temperature rises and thermal deformations in a 3D double-layered thin film consisting of a gold layer on a chromium padding layer with dimensions $100 \mu \mathrm{m} \times 100 \mu \mathrm{m} \times 0.1 \mu \mathrm{m}$, and in a $3 \mathrm{D}$ double-layered micro sphere consisting of a gold layer on a chromium padding layer with a radius of $0.05 \mu \mathrm{m}$ for each layer, respectively. The thermophysical properties for gold and chromium are listed in Table 1 [1, 5, 17]. We assumed that the laser was focused on the center of the top surface of the thin film and that it irradiated the top surface $\left(0 \leq \varphi \leq \frac{\pi}{4}\right)$ of the sphere, respectively. Three different values of laser fluences $(J=500$ $\mathrm{J} / \mathrm{m}^{2}, 1000 \mathrm{~J} / \mathrm{m}^{2}$ and $2000 \mathrm{~J} / \mathrm{m}^{2}$ ) were chosen to study the hot electron blast force. Three meshes of $20 \times 20 \times 60,20 \times 20 \times 80,20 \times 20 \times 100$ for each layer in $(x, y, z)$ for the thin film and three meshes of $60 \times 20 \times 20,80 \times 20 \times 20,100 \times 20 \times 20$ for each layer in $(r, \theta, \varphi)$ for the micro sphere were chosen in order to test the convergence of the scheme. The time increment was chosen to be $0.005 \mathrm{ps}$ and $T_{0}$ was set to be $300 \mathrm{~K}$. The convergence criteria were chosen to be $\xi_{1}=10^{-8}$ for temperature and $\xi_{1}=10^{-16}$ for deformation.

Fig. (3a) shows the changes in electron temperature $\left(\Delta T_{e} /\left(\Delta T_{e}\right)_{\max }\right) \quad$ at the center $\quad\left(x_{\text {center }}=50 \mu \mathrm{m}\right.$, $y_{\text {center }}=50 \mu \mathrm{m}$ and $z=0 \mu \mathrm{m}$ ) of the thin film and at the top point $(L, 0,0)$ of the sphere with a laser fluence of $J=500 \mathrm{~J} / \mathrm{m}^{2}$, respectively. The maximum temperature rise of Te (i.e., $\left.\left(\Delta T_{e}\right)_{\max }\right)$ is about $3765 \mathrm{~K}$, which is close to the $3727 \mathrm{~K}$ obtained by Tzou et al. [1]. It can be seen from this figure that there is a slight difference between the thin film and the sphere. This is probably because of the different geometries. Fig. (3b) shows the displacement $(w)$ at the center $\left(x_{\text {center }}, y_{\text {center }}, 0\right)$ of the thin film and the displacement $\left(u_{r}\right)$ at the top point $(L, 0,0)$ of the sphere versus time, respectively. The negative value of displacement $(w)$ indicates that the thin film at the center $\left(x_{\text {center }}, y_{\text {center }}, 0\right)$ is expanding along the negative $z$ direction, while the positive value of displacement $\left(u_{r}\right)$ implies that the sphere at the top point 


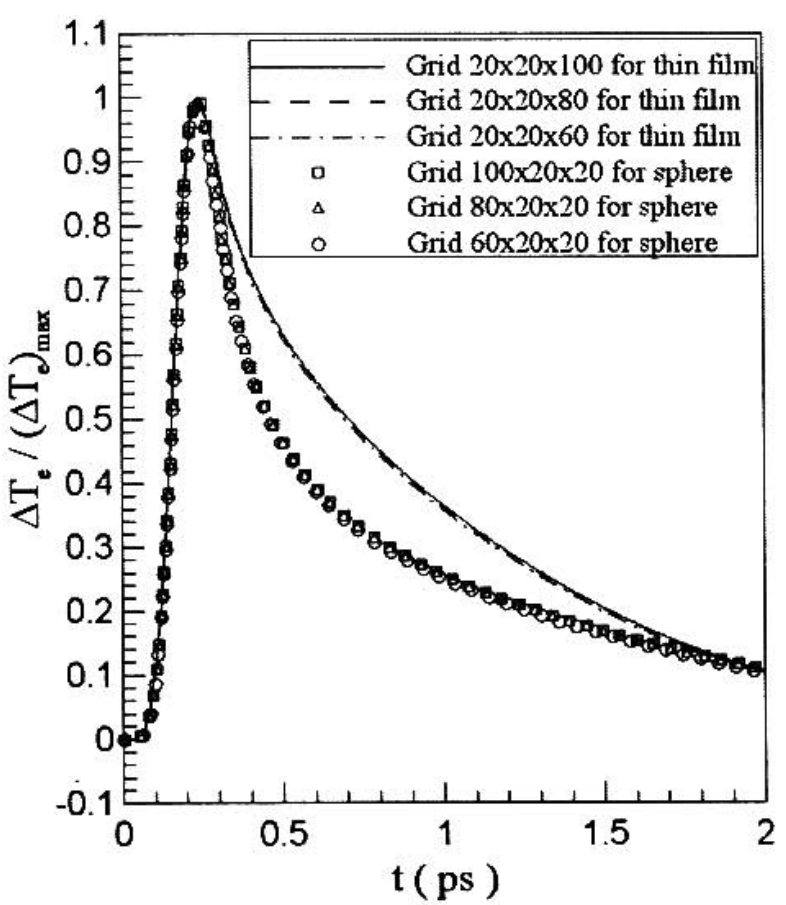

(a)

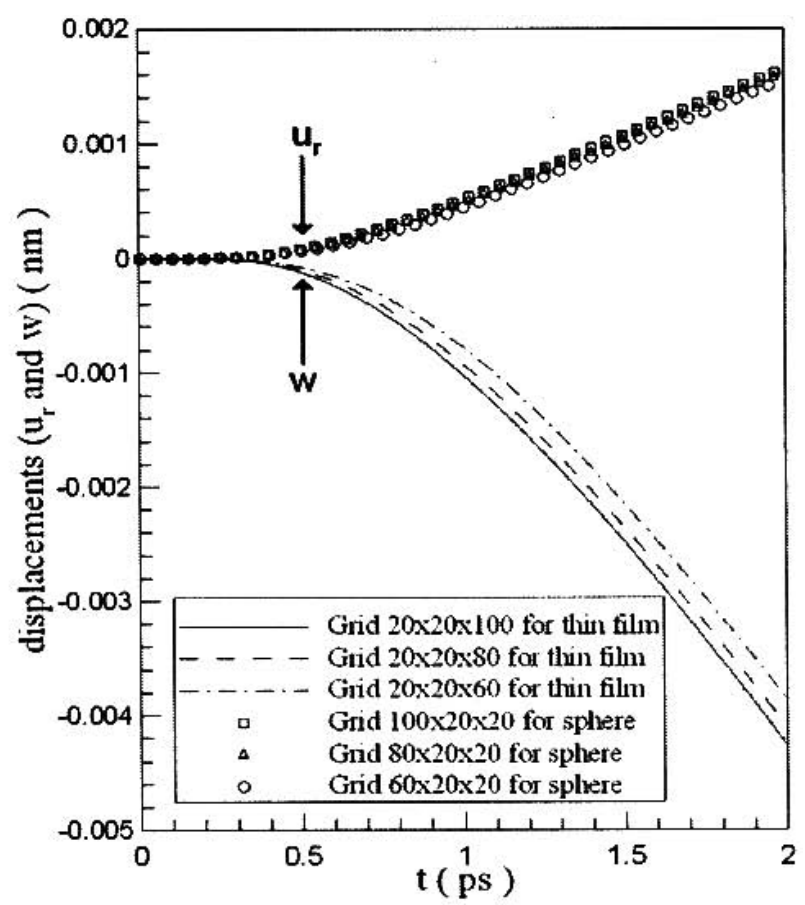

(b)

Fig. (3a). Change in electron temperature and (b) displacements at the center of top surface of thin film and at the top point of sphere versus time with a laser fluence $(J)$ of $500 \mathrm{~J} / \mathrm{m}^{2}$. The $w$ is the displacement at $\left(\mathrm{x}_{\text {center }}, \mathrm{y}_{\text {center }}, 0\right)$ of thin film and $u_{r}$ is the displacement at the top point $(L, 0,0)$ of sphere, respectively.

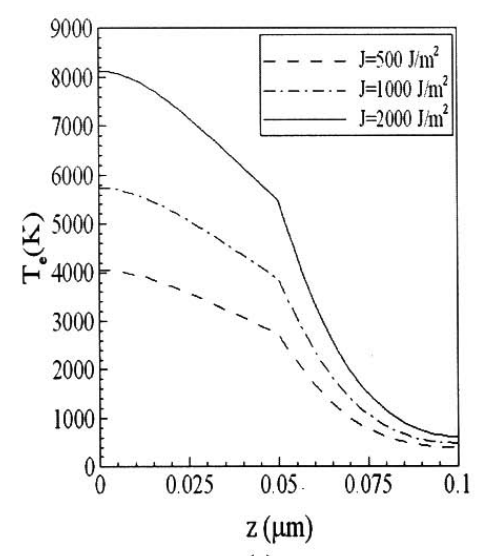

(a)

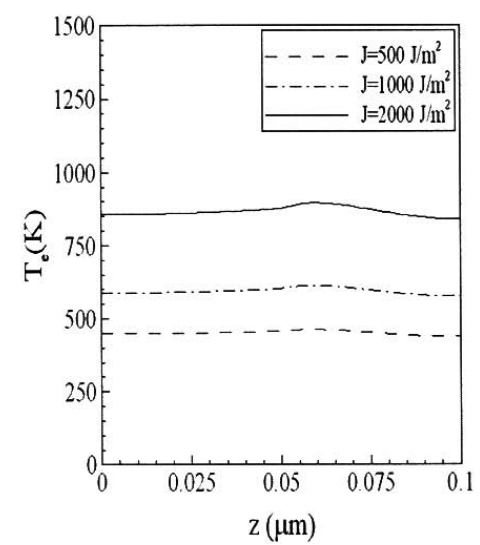

(c)

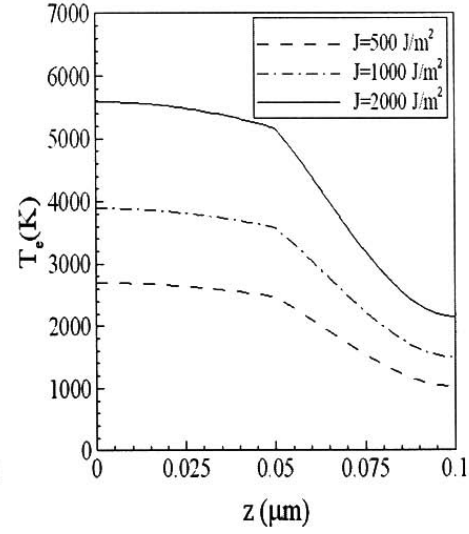

(b)

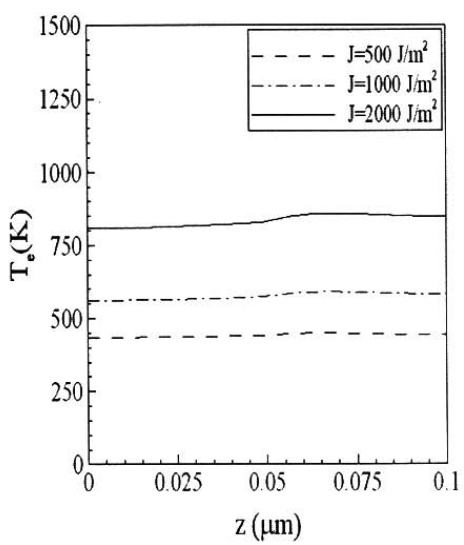

(d)

Fig. (4). Electron temperature profiles along $z$ at $\left(\mathrm{x}_{\text {center, }} \mathrm{y}_{\text {center }}\right)$ at different times (a) $t=0.25 \mathrm{ps,} \mathrm{(b)} t=0.5 \mathrm{ps,} \mathrm{(c)} t=10 \mathrm{ps,} \mathrm{and} \mathrm{(d)} t=$ 20 ps with a mesh of $20 \times 20 \times 80$ and three different laser fluences $(J)$ of $500 \mathrm{~J} / \mathrm{m}^{2}, 1000 \mathrm{~J} / \mathrm{m}^{2}$ and $2000 \mathrm{~J} / \mathrm{m}^{2}$. 


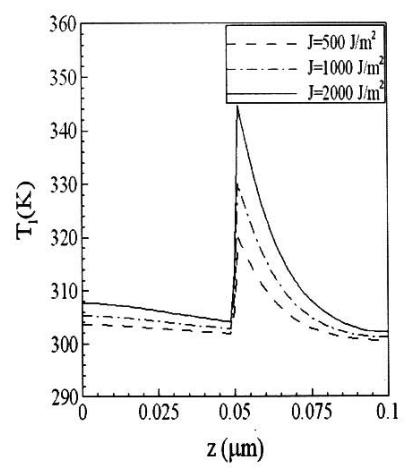

(a)

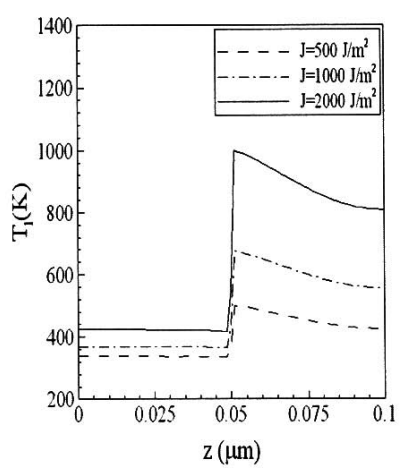

(c)

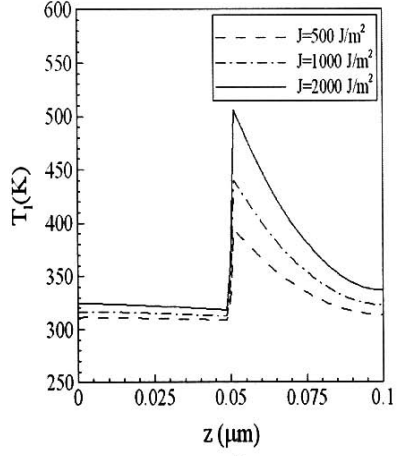

(b)

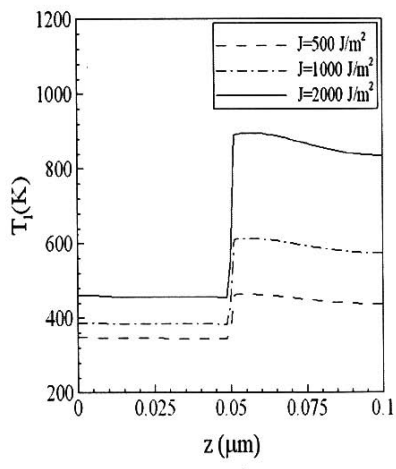

(d)

Fig. (5). Lattice temperature profiles along $z$ at $\left(\mathrm{x}_{\text {center, }} \mathrm{y}_{\text {center }}\right)$ at different times (a) $t=0.25 \mathrm{ps,} \mathrm{(b)} t=0.5 \mathrm{ps,} \mathrm{(c)} t=10 \mathrm{ps,} \mathrm{and} \mathrm{(d)} t=$ 20 ps with a mesh of $20 \times 20 \times 80$ and three different laser fluences $(J)$ of $500 \mathrm{~J} / \mathrm{m}^{2}, 1000 \mathrm{~J} / \mathrm{m}^{2}$ and $2000 \mathrm{~J} / \mathrm{m}^{2}$.

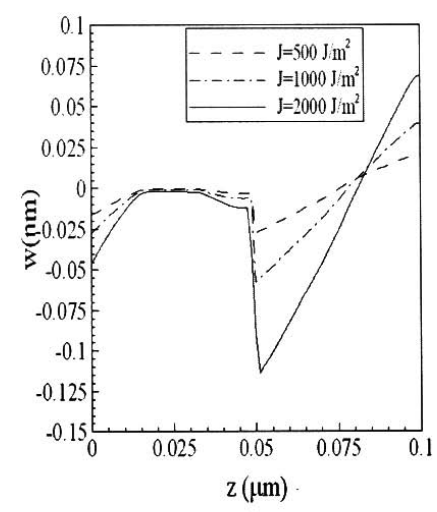

(a)

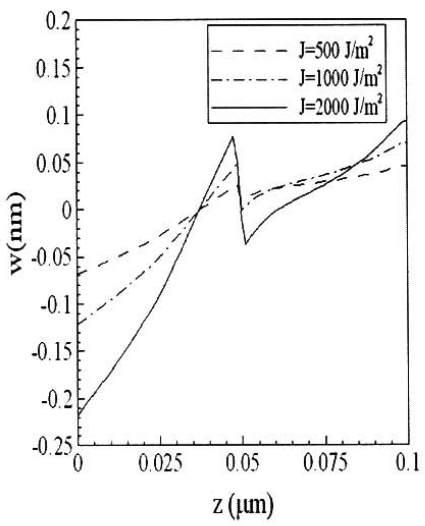

(c)

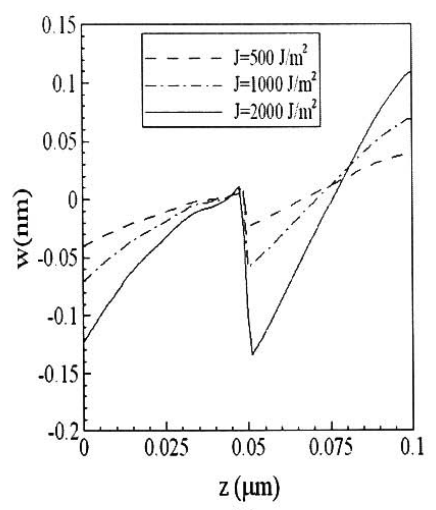

(b)

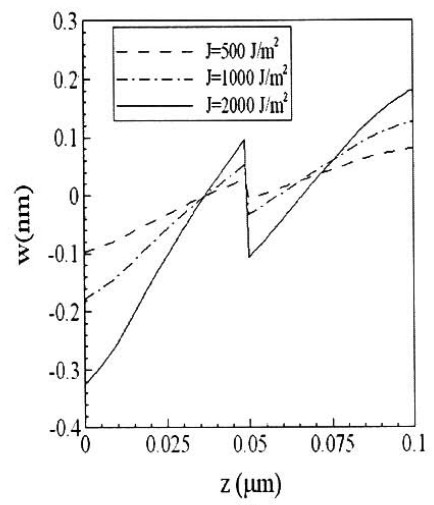

(d)

Fig. (6). Displacement (w) profiles along $z$ at $\left(\mathrm{x}_{\text {center, }} \mathrm{y}_{\text {center }}\right)$ at different times (a) $t=5 \mathrm{ps,} \mathrm{(b)} t=10 \mathrm{ps,} \mathrm{(c)} t=15 \mathrm{ps,} \mathrm{and} \mathrm{(d)} t=20 \mathrm{ps}$ with a mesh of $20 \times 20 \times 80$ and three different laser fluences $(J)$ of $500 \mathrm{~J} / \mathrm{m}^{2}, 1000 \mathrm{~J} / \mathrm{m}^{2}$ and $2000 \mathrm{~J} / \mathrm{m}^{2}$. 


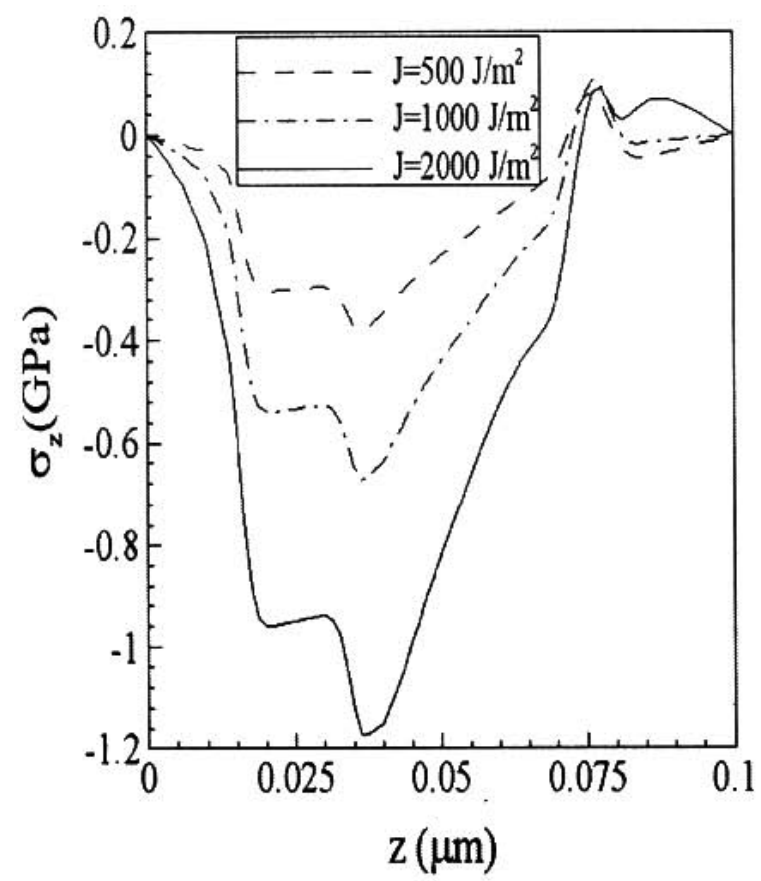

(a)

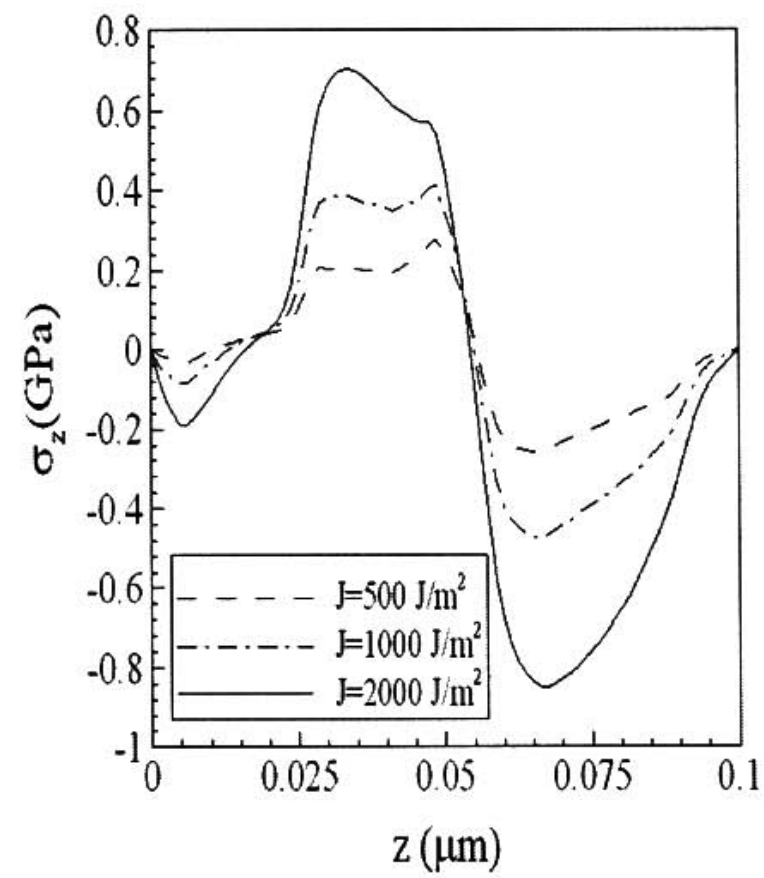

(c)

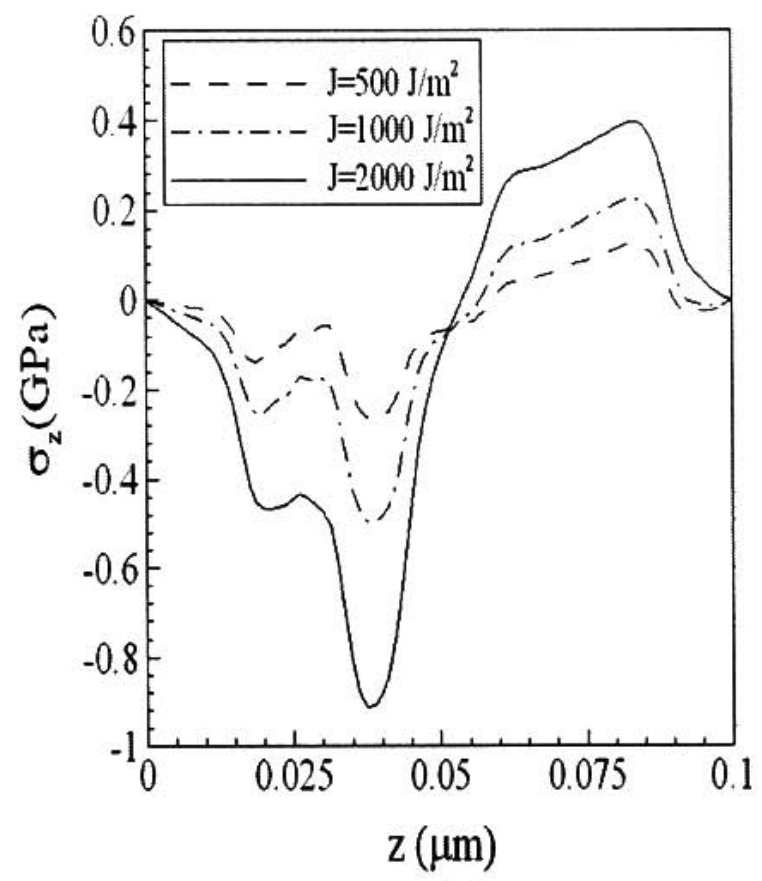

(b)

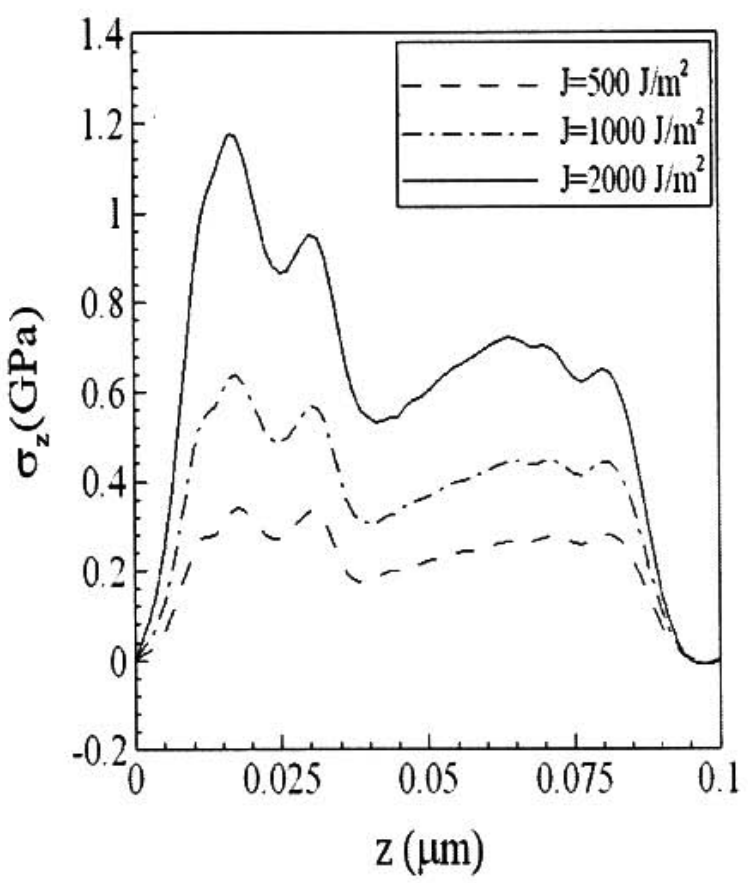

(d)

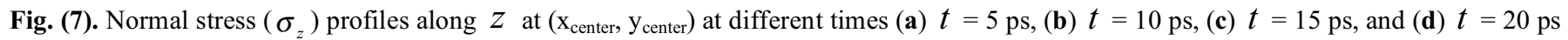
with a mesh of $20 \times 20 \times 80$ and three different laser fluences $(J)$ of $500 \mathrm{~J} / \mathrm{m}^{2}, 1000 \mathrm{~J} / \mathrm{m}^{2}$ and $2000 \mathrm{~J} / \mathrm{m}^{2}$.

$(L, 0,0)$ is expanding along the positive $r$ direction. It can be seen from both figures that the mesh size had no significant effect on the solution and hence the solution is convergent.

Figs. (4 and 5) show electron temperature and lattice temperature of the thin film along $z$ at $\left(x_{\text {center }}, y_{\text {center }}\right)$ with three different laser fluences $\left(J=500 \mathrm{~J} / \mathrm{m}^{2}, 1000 \mathrm{~J} / \mathrm{m}^{2}\right.$ and $2000 \mathrm{~J} / \mathrm{m}^{2}$ ) at different times (a) $\mathrm{t}=0.25 \mathrm{ps}$, (b) $\mathrm{t}=0.5 \mathrm{ps}$, (c) $\mathrm{t}=10 \mathrm{ps}$, and (d) $\mathrm{t}=20 \mathrm{ps}$, respectively. It can be seen from Fig. (4) that the electron temperature is in maximum at $t=$ $0.25 \mathrm{ps}$, then it decays with time and it is almost uniform at $\mathrm{t}$ $=20 \mathrm{ps}$ along the thickness direction. On the other hand, Fig. (5) shows that the lattice temperature increases gradually 


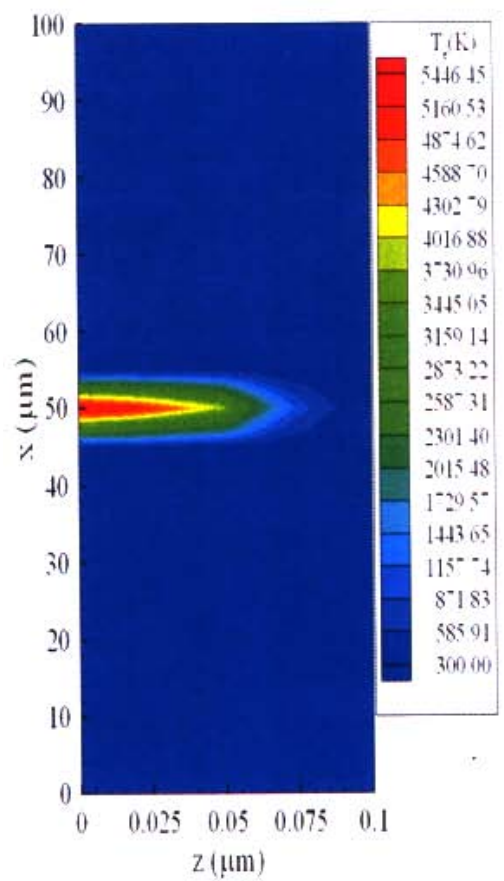

(a)

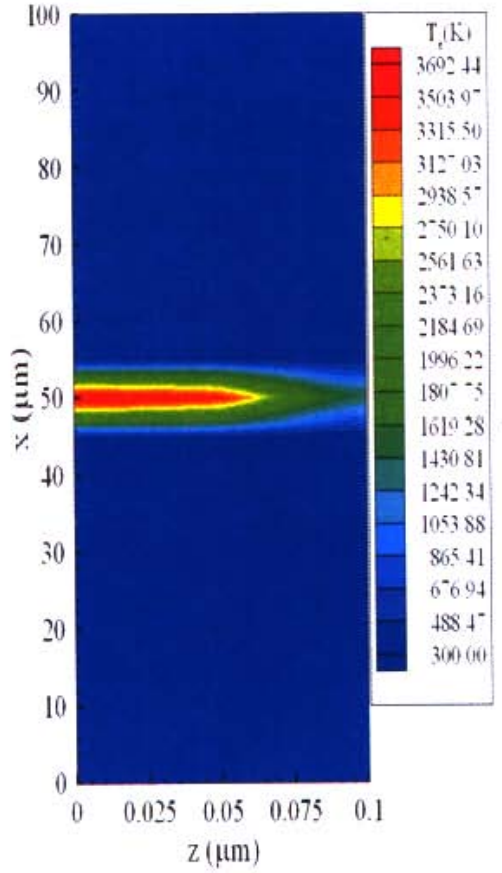

(b)

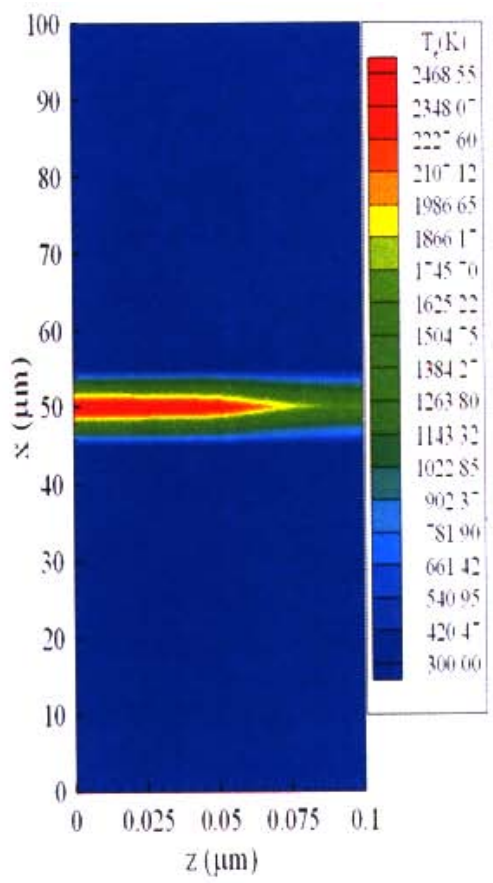

(c)

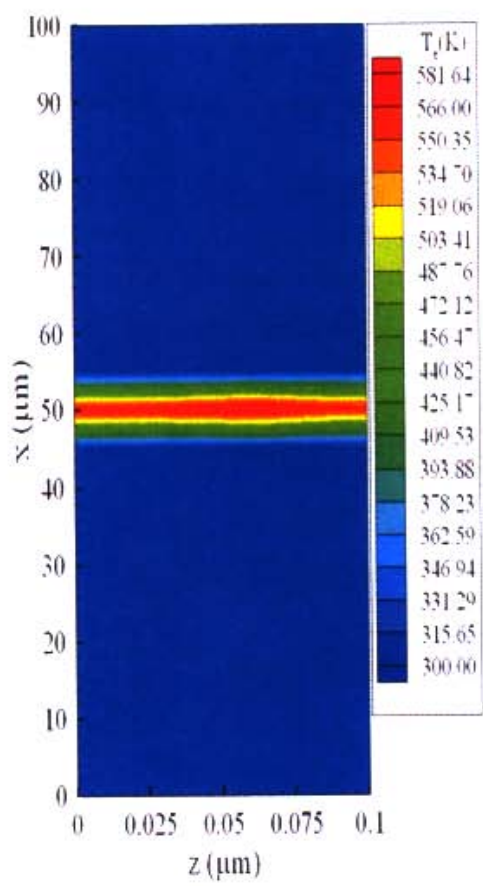

(d)

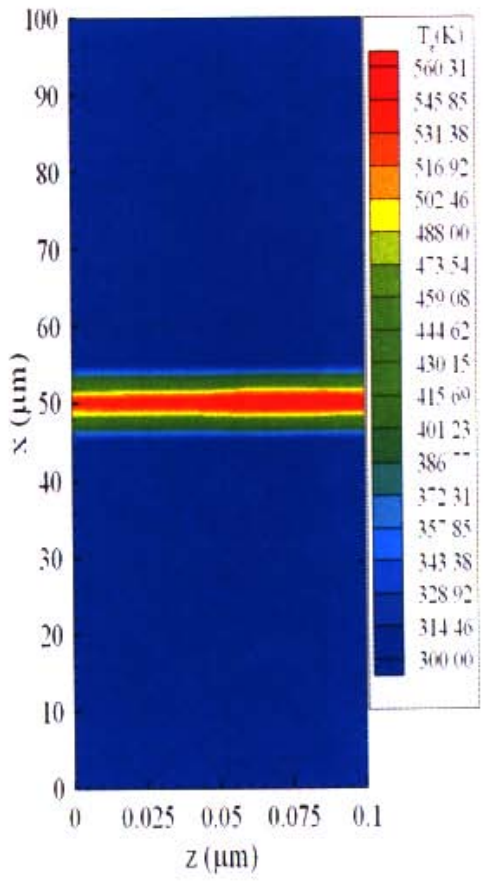

(e)

Fig. (8). Contours of electron temperature distributions in the cross section of $y=50 \mu \mathrm{m}$ at different times (a) $t=0.25 \mathrm{ps}$, (b) $t=0.5 \mathrm{ps,}$ (c) $t=1 \mathrm{ps,} \mathrm{(d)} t=10 \mathrm{ps}$, and (e) $t=20 \mathrm{ps}$ with a mesh of $20 \times 20 \times 80$ and a laser fluence $(J)$ of $1000 \mathrm{~J} / \mathrm{m}^{2}$.

with time in both gold and chromium layers, due to constant heating of acoustic phonons by electrons. Since the heat is transferred from the gold layer to the chromium layer and the conductivity of chromium is smaller than that of gold, the lattice temperature increases drastically across the interface. A clear discontinuity of the temperature gradient at the interface can be seen in Fig. (5), which is the same prediction as was obtained in $[1,11]$. The difference of electron and lattice temperatures in Figs. (4 and 5) gives a strong flavor of nonequilibrium heating during the picosecond transient.

Fig. (6) shows the displacement $(w)$ of the thin film along $z$ at $\left(x_{\text {center }}, y_{\text {center }}\right)$ at different times (a) $\mathrm{t}=5 \mathrm{ps}$, (b) 


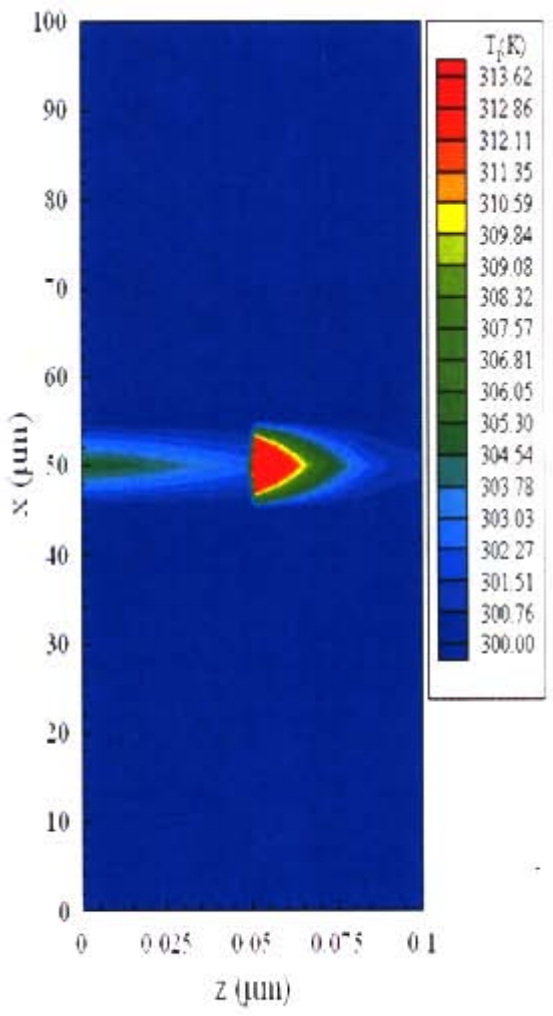

(a)

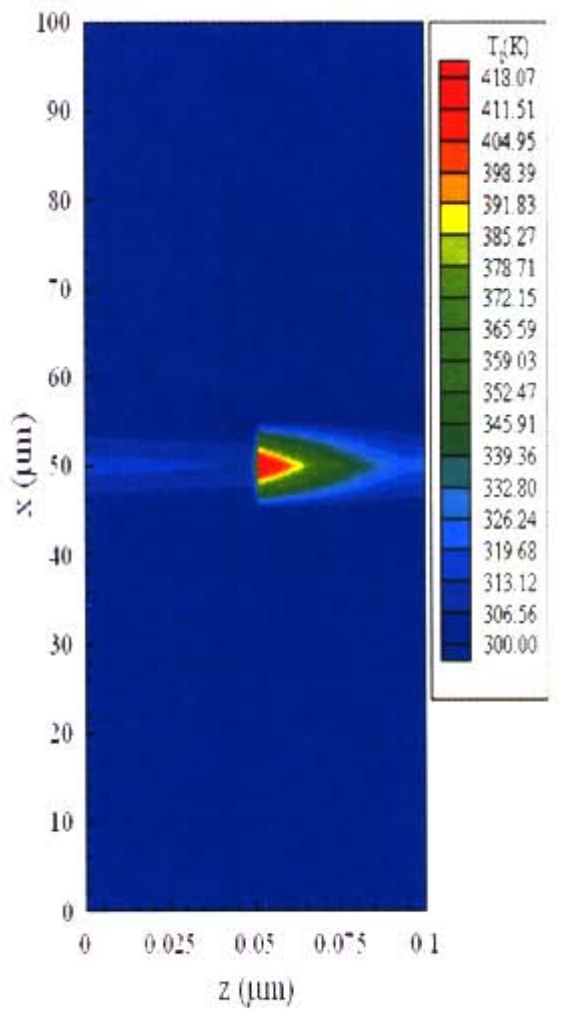

(b)

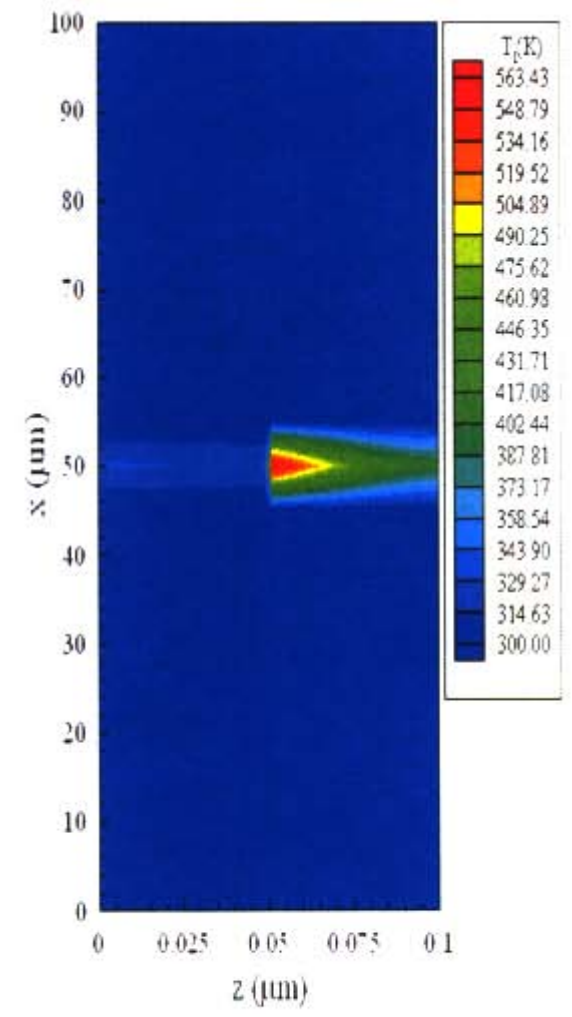

(c)

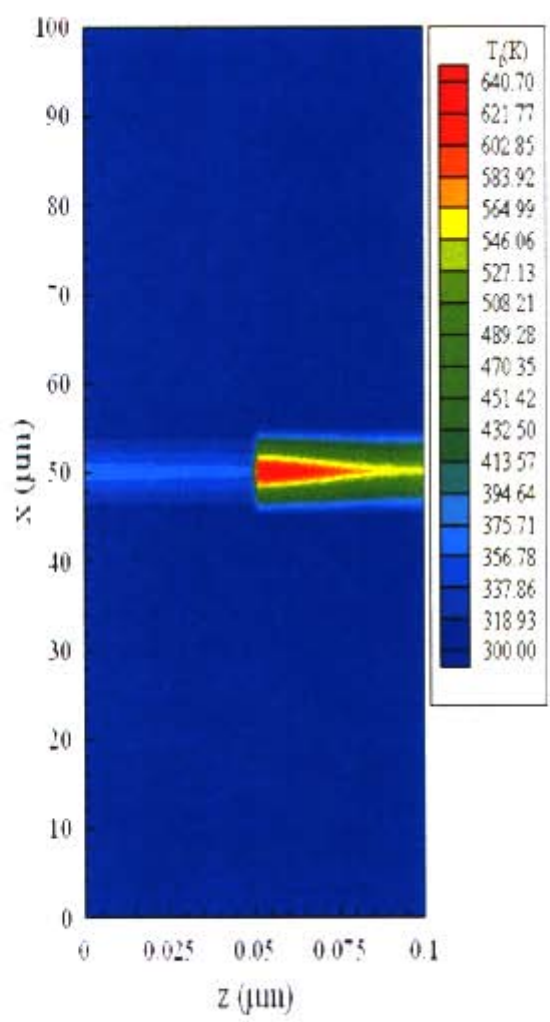

(d)

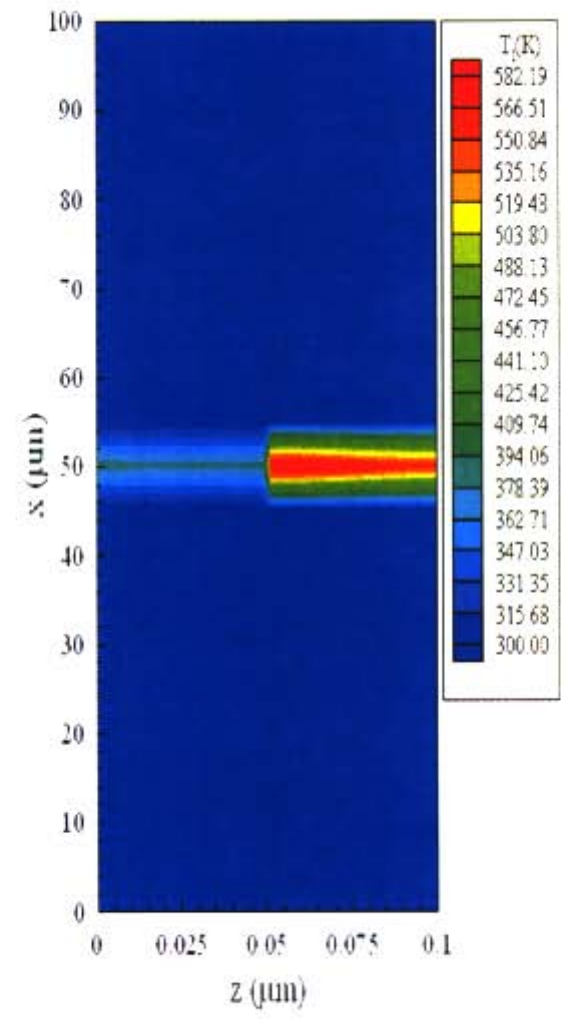

(e)

Fig. (9). Contours of lattice temperature distributions in the cross section of $y=50 \mu \mathrm{m}$ at different times (a) $t=0.25 \mathrm{ps,} \mathrm{(b)} t=0.5 \mathrm{ps}$, (c) $t=1 \mathrm{ps}$, (d) $t=10 \mathrm{ps}$, and (e) $t=20 \mathrm{ps}$ with a mesh of $20 \times 20 \times 80$ and a laser fluence $(J)$ of $1000 \mathrm{~J} / \mathrm{m}^{2}$. 


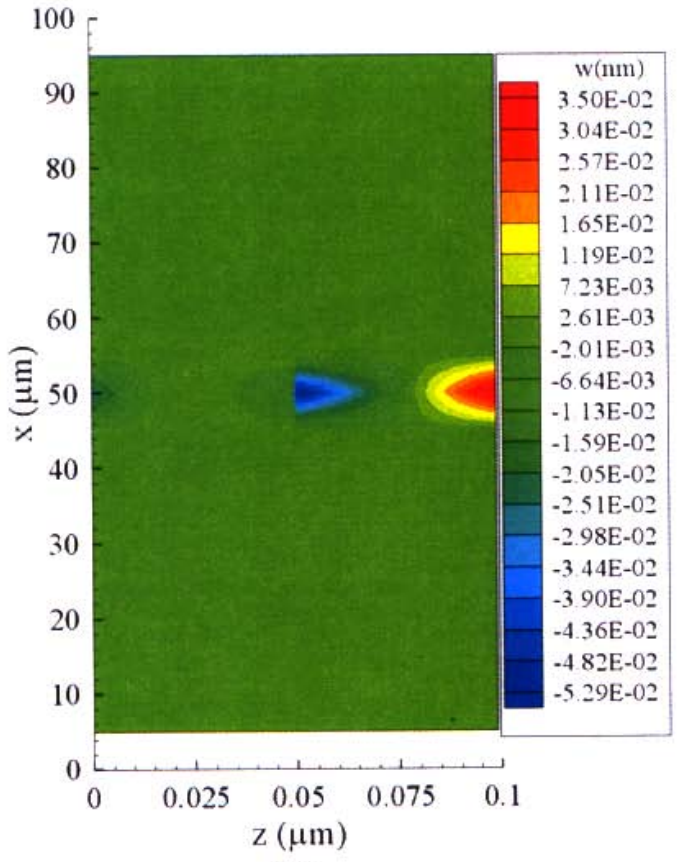

(a)

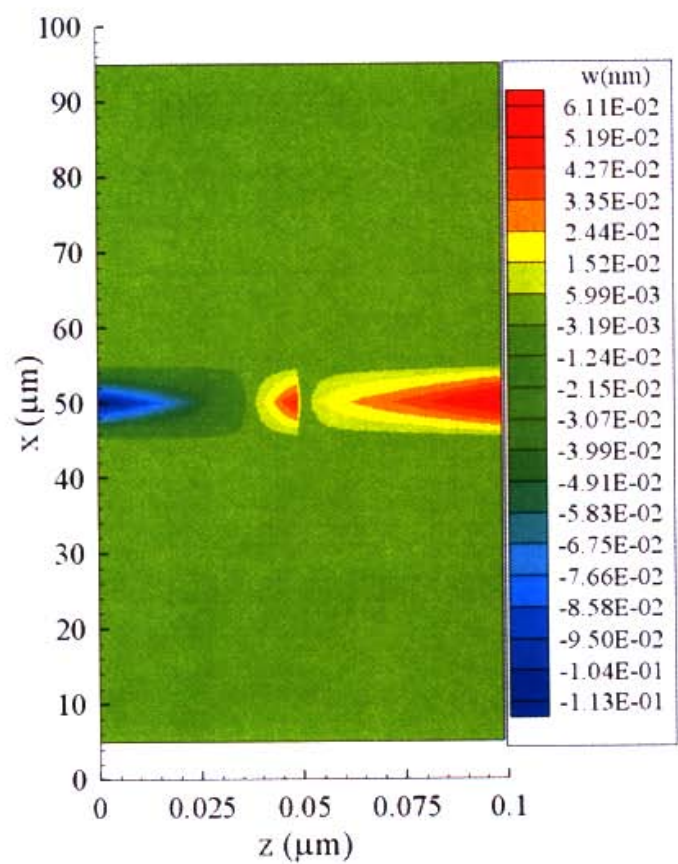

(c)

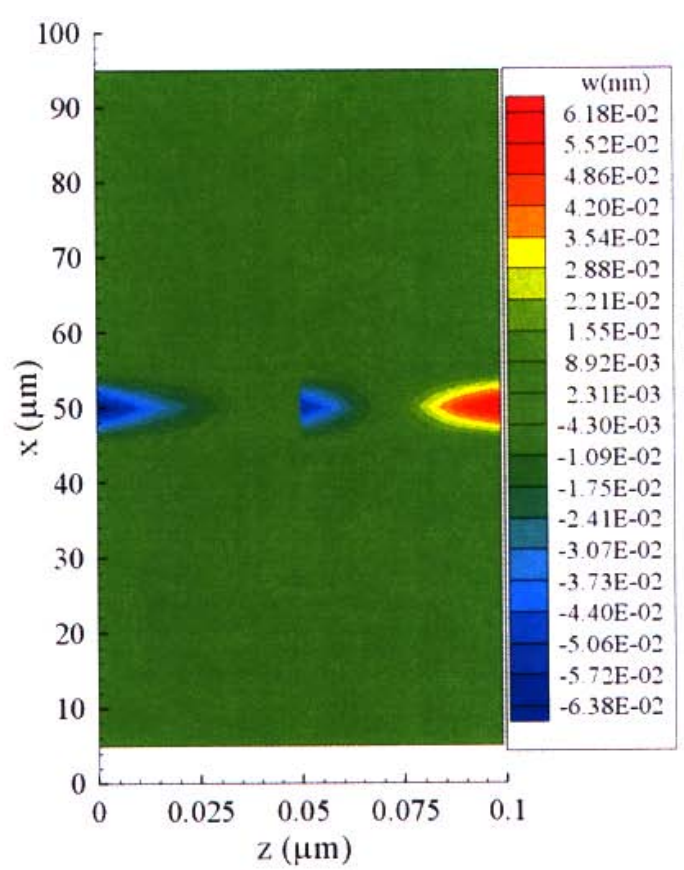

(b)

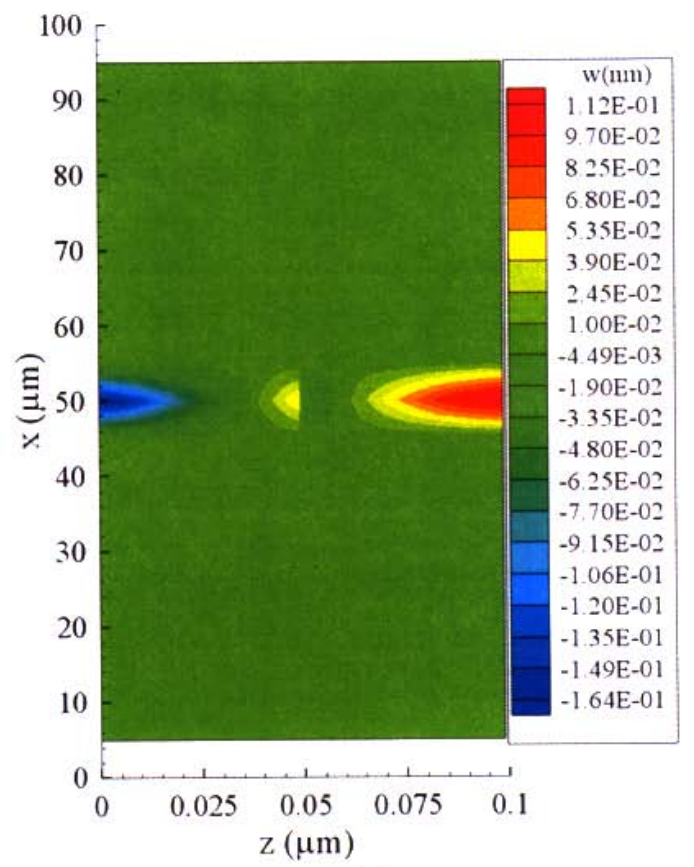

(d)

Fig. (10). Contours of displacement (w) distributions in the cross section of $y=50 \mu \mathrm{m}$ at different times (a) $t=5 \mathrm{ps,} \mathrm{(b)} t=10 \mathrm{ps,} \mathrm{(c)} t=$ $15 \mathrm{ps}$, and (d) $t=20 \mathrm{ps}$ with a mesh of $20 \times 20 \times 80$ and a laser fluence $(J)$ of $1000 \mathrm{~J} / \mathrm{m}^{2}$.

$\mathrm{t}=10 \mathrm{ps}$, (c) $\mathrm{t}=15 \mathrm{ps}$, and (d) $\mathrm{t}=20$ ps with a mesh of $20 \times 20 \times 80$ and three different laser fluences

$\left(J=500 \mathrm{~J} / \mathrm{m}^{2}, 1000 \mathrm{~J} / \mathrm{m}^{2}\right.$ and $\left.2000 \mathrm{~J} / \mathrm{m}^{2}\right)$. It can been seen that the displacement $w$, particularly at $\mathrm{t}=20 \mathrm{ps}$, changes from negative to positive for each layer along the thickness direction. The negative value indicates that the displacement moves in the negative $z$ direction, while the positive value implies that it moves in the positive $z$ direction. From this figure, one may see that the film is expanding. At $\mathrm{t}=10 \mathrm{ps}$ and $20 \mathrm{ps}$, the displacement shows a clear alteration across the interface, implying that both layers push each other and the bond between these two layers could be damaged under high intensity laser irradiation.

Fig. (7) shows the normal stress $\sigma_{z}$ along $z$ at $\left(x_{\text {center }}, y_{\text {center }}\right)$ at different times (a) $\mathrm{t}=5 \mathrm{ps}$, (b) $\mathrm{t}=10 \mathrm{ps}$, (c) $\mathrm{t}=15 \mathrm{ps}$, and (d) $\mathrm{t}=20 \mathrm{ps}$ with a mesh of $20 \times 20 \times 80$ and three different laser fluences $\left(J=500 \mathrm{~J} / \mathrm{m}^{2}\right.$, $1000 \mathrm{~J} / \mathrm{m}^{2}$ and $2000 \mathrm{~J} / \mathrm{m}^{2}$ ). In our experience, the conventional finite difference method produces local oscillations in the normal stress $\sigma_{z}$ (see Fig. (5) in [2]). It can be seen from 


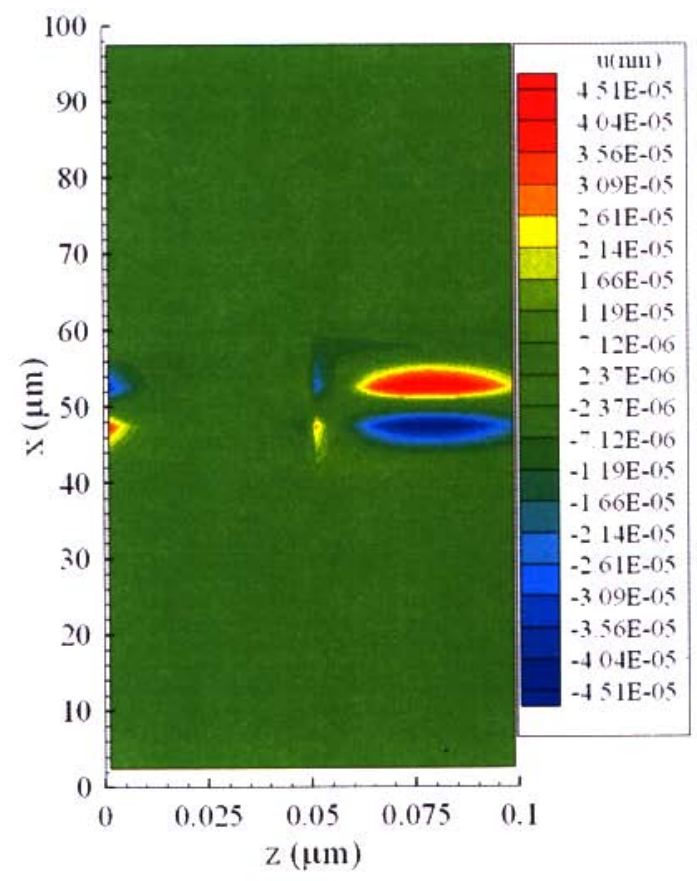

(a)

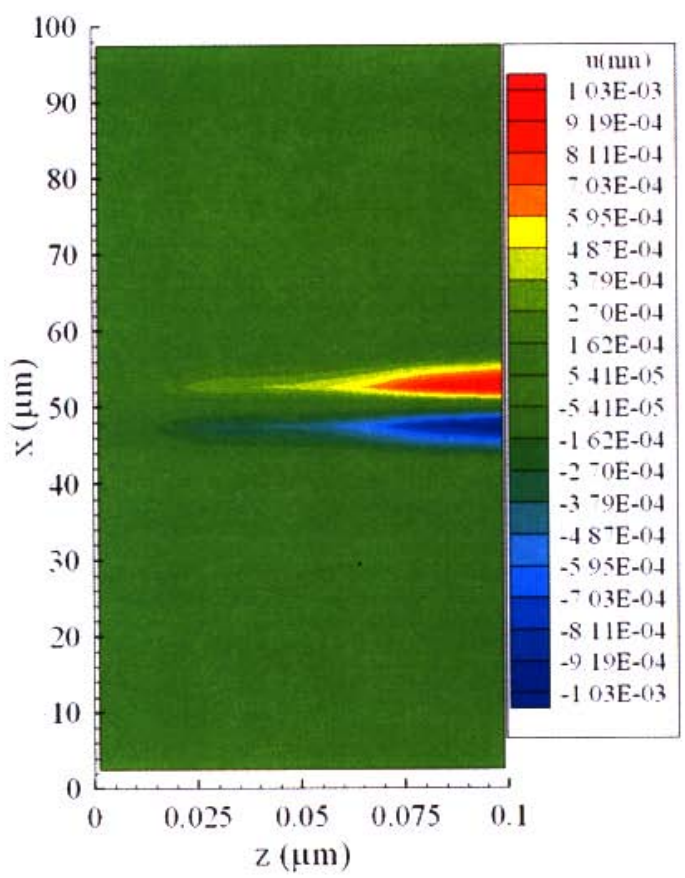

(c)

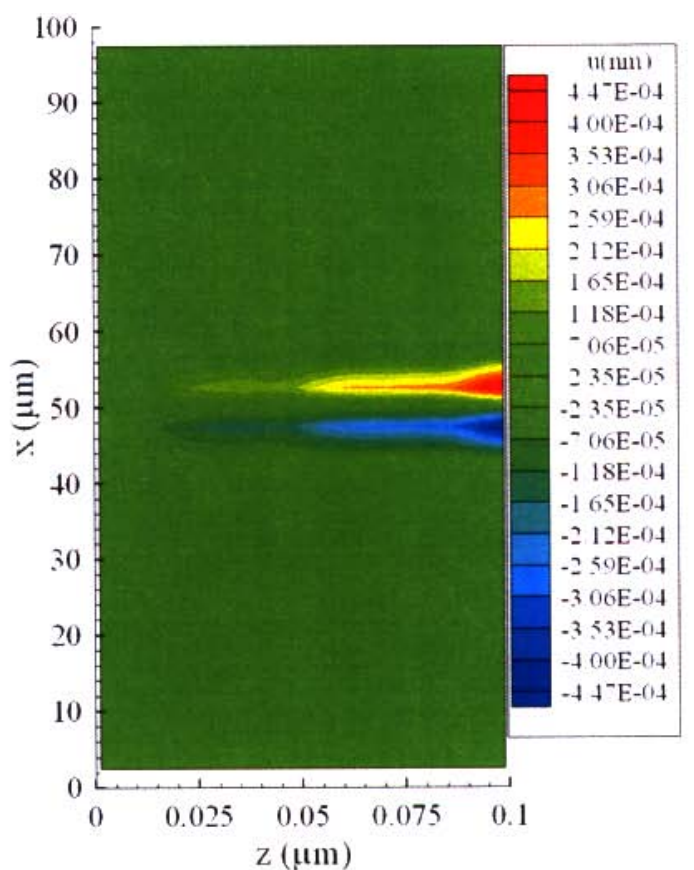

(b)

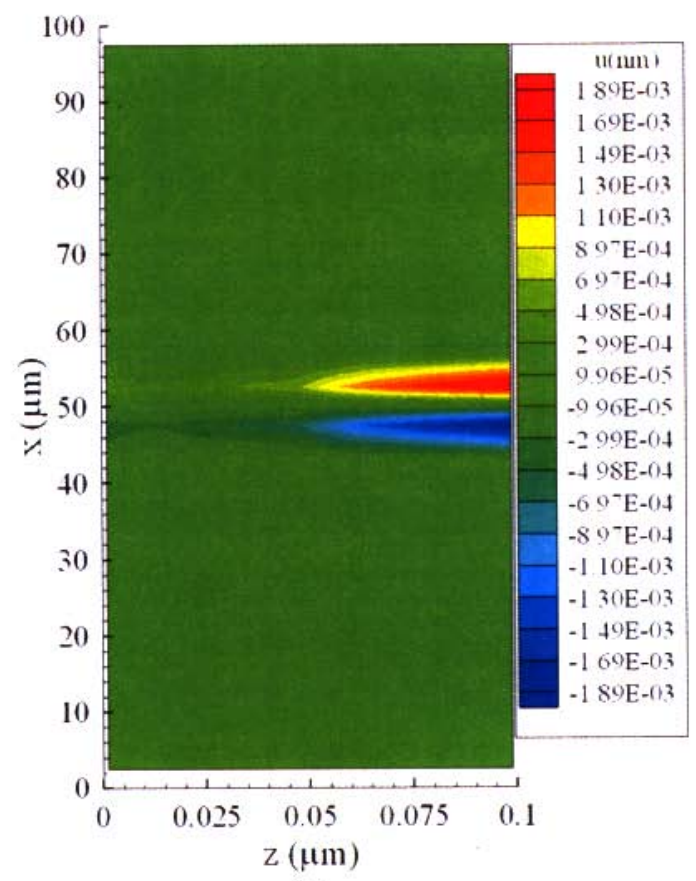

(d)

Fig. (11). Contours of displacement $(u)$ distributions in the cross section of $y=50 \mu \mathrm{m}$ at different times (a) $t=5 \mathrm{ps,} \mathrm{(b)} t=10 \mathrm{ps}$, (c) $t=$ $15 \mathrm{ps}$, and (d) $t=20 \mathrm{ps}$ with a mesh of $20 \times 20 \times 80$ and a laser fluence $(J)$ of $1000 \mathrm{~J} / \mathrm{m}^{2}$.

Fig. (7) that the curve of $\sigma_{z}$ is smooth and does not appear to have local oscillations, implying that our method prevents the appearance of non-physical oscillations in the solution.

Figs. (8-12) were plotted based on the results obtained with a mesh of $20 \times 20 \times 80$ and with a laser fluence of $J=1000 \mathrm{~J} / \mathrm{m}^{2}$. Figs. (8 and 9) show contours of the electron temperature distribution and the lattice temperature distribution in the cross section of $y=y_{\text {center }}$ at different times (a) $\mathrm{t}=0.25 \mathrm{ps}$, (b) $\mathrm{t}=0.5 \mathrm{ps}$, (c) $\mathrm{t}=1 \mathrm{ps}$, (d) $\mathrm{t}=10 \mathrm{ps}$, and (e) $\mathrm{t}=20 \mathrm{ps}$, respectively. It can be seen from both figures that the heat is mainly transferred along the $z$ direction. This result confirms the fact that the femtosecond lasers are an ideal candidate for precise thermal processing of functional nanophase materials. Fig. (9) also shows that there is a clear difference between the lattice temperatures in these two layers, because of the different conductivities. Figs. (10-12) show contours of displacements $(u, v, w)$ in the cross section of $y=y_{\text {center }}$ at different times (a) $\mathrm{t}=5 \mathrm{ps}$, (b) $\mathrm{t}=10 \mathrm{ps}$, (c) 


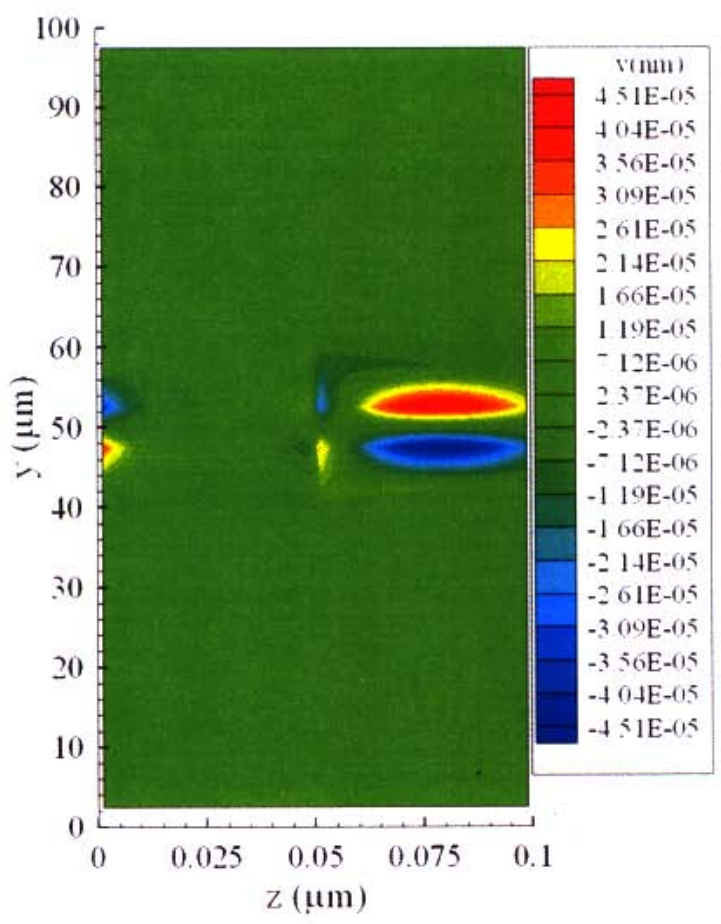

(a)

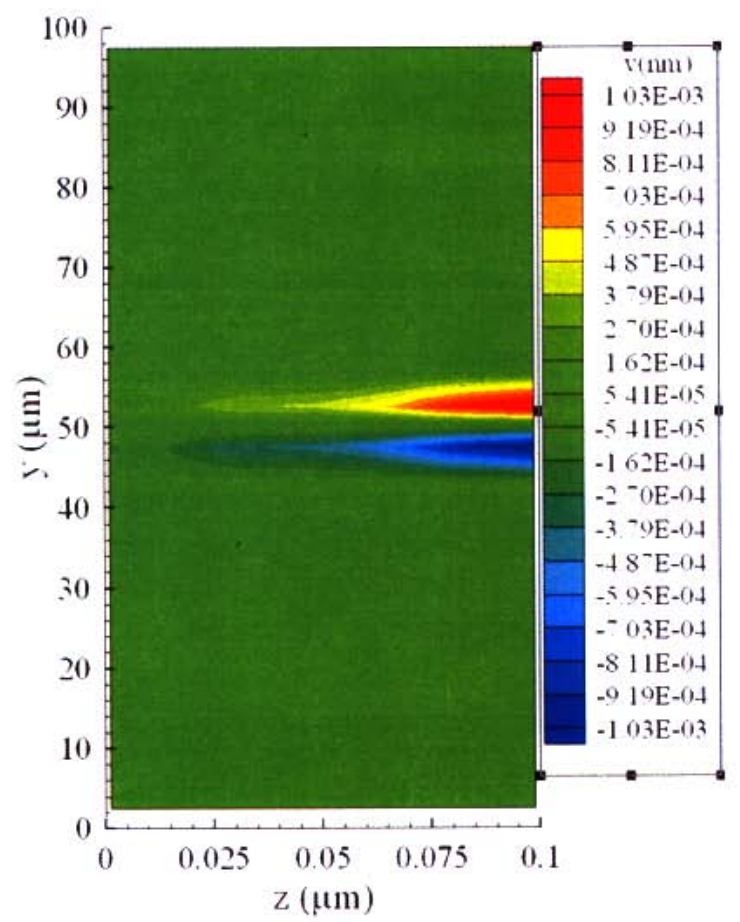

(c)

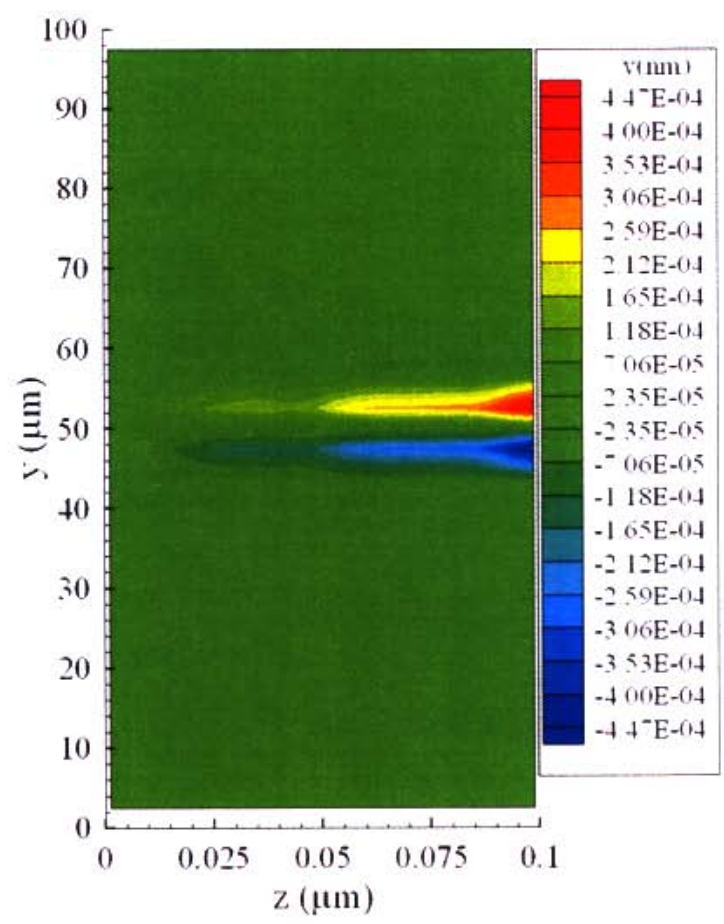

(b)

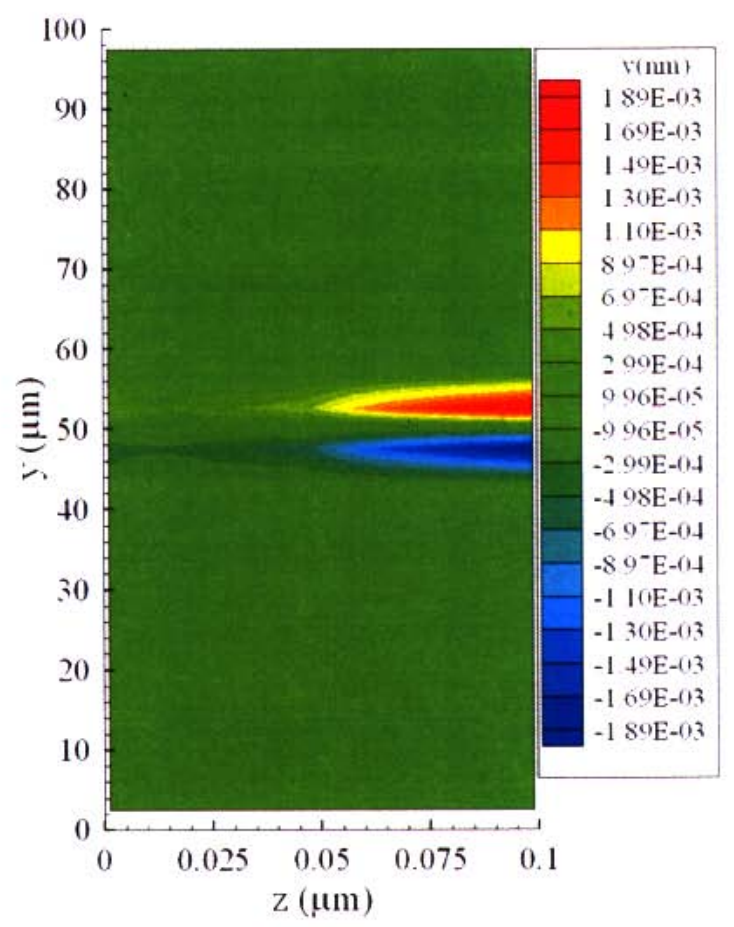

(d)

Fig. (12). Contours of displacement (v) distributions in the cross section of $y=50 \mu \mathrm{m}$ at different times (a) $t=5 \mathrm{ps,} \mathrm{(b)} t=10 \mathrm{ps,}(\mathbf{c}) t=$ $15 \mathrm{ps}$, and (d) $t=20 \mathrm{ps}$ with a mesh of $20 \times 20 \times 80$ and a laser fluence $(J)$ of $1000 \mathrm{~J} / \mathrm{m}^{2}$.

$\mathrm{t}=15 \mathrm{ps}$, and (d) $\mathrm{t}=20 \mathrm{ps}$, respectively. It can be seen from Figs. (10-12) that the central part of the film is expanding because displacements change from negative to positive along the center line in the $z$ direction, and along the $x$ and $y$ directions, respectively.

We now turn our attention to the sphere. Figs. (13) and (14) show profiles of the electron temperature and the lattice temperature along the diameter at $\varphi=0$ and $\varphi=\pi$ with three different laser $\left(J=500 \mathrm{~J} / \mathrm{m}^{2}, 1000 \mathrm{~J} / \mathrm{m}^{2}\right.$ and $\left.2000 \mathrm{~J} / \mathrm{m}^{2}\right)$ at different times(a) $\mathrm{t}=0.25 \mathrm{ps}$, (b) $\mathrm{t}=0.5 \mathrm{ps}$, (c) $\mathrm{t}=10 \mathrm{ps}$, and (d) $t=20 \mathrm{ps}$, respectively. Again, it can be seen from Fig. (13) that the heat is transferred from the gold layer to the chromium layer, and the lattice temperature increases drastically across the interface. A clear discontinuity of the temperature gradient at the interface is also seen in Fig. (14). 


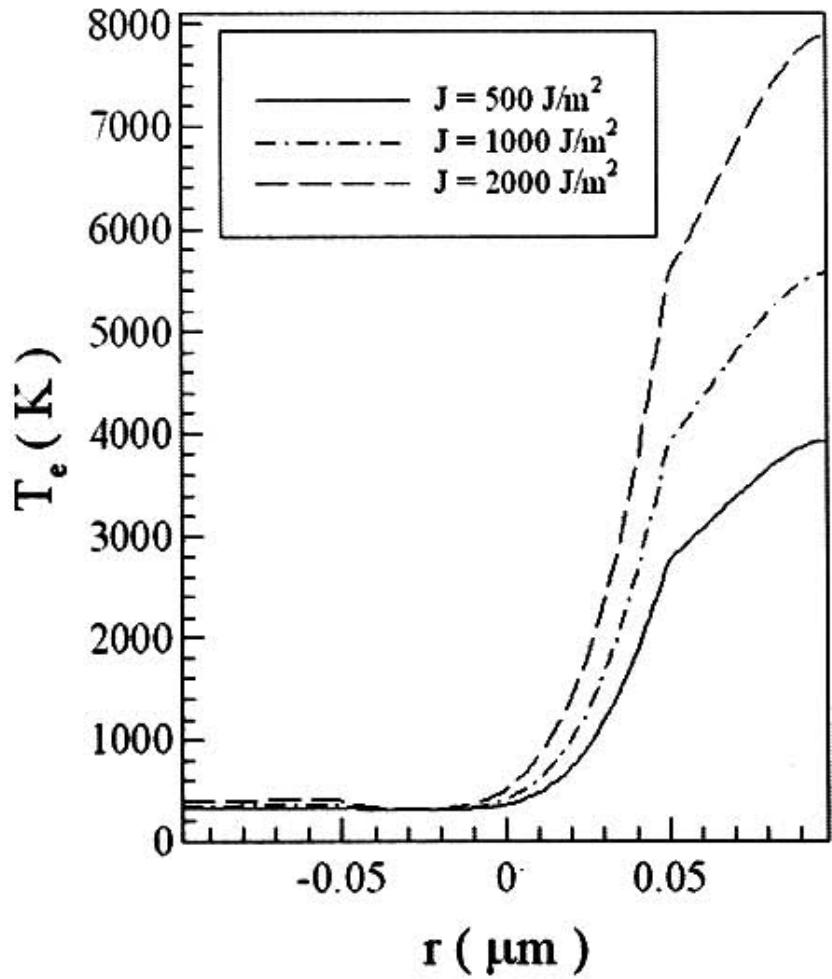

(a)

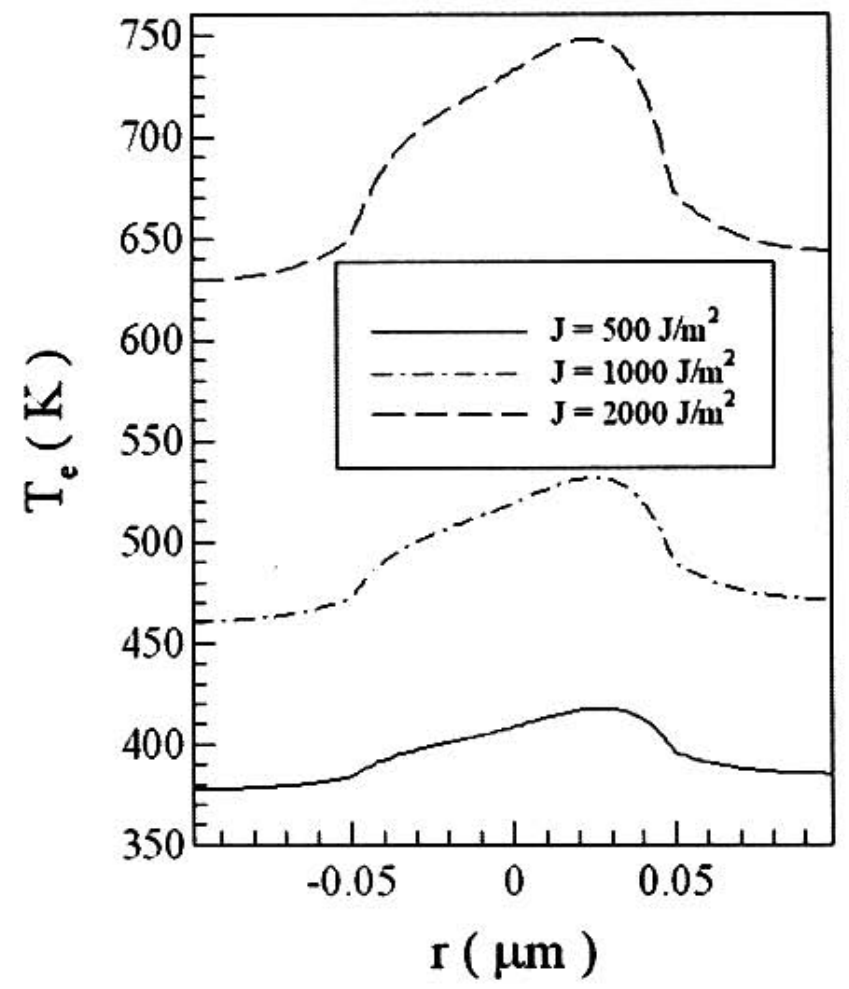

(c)

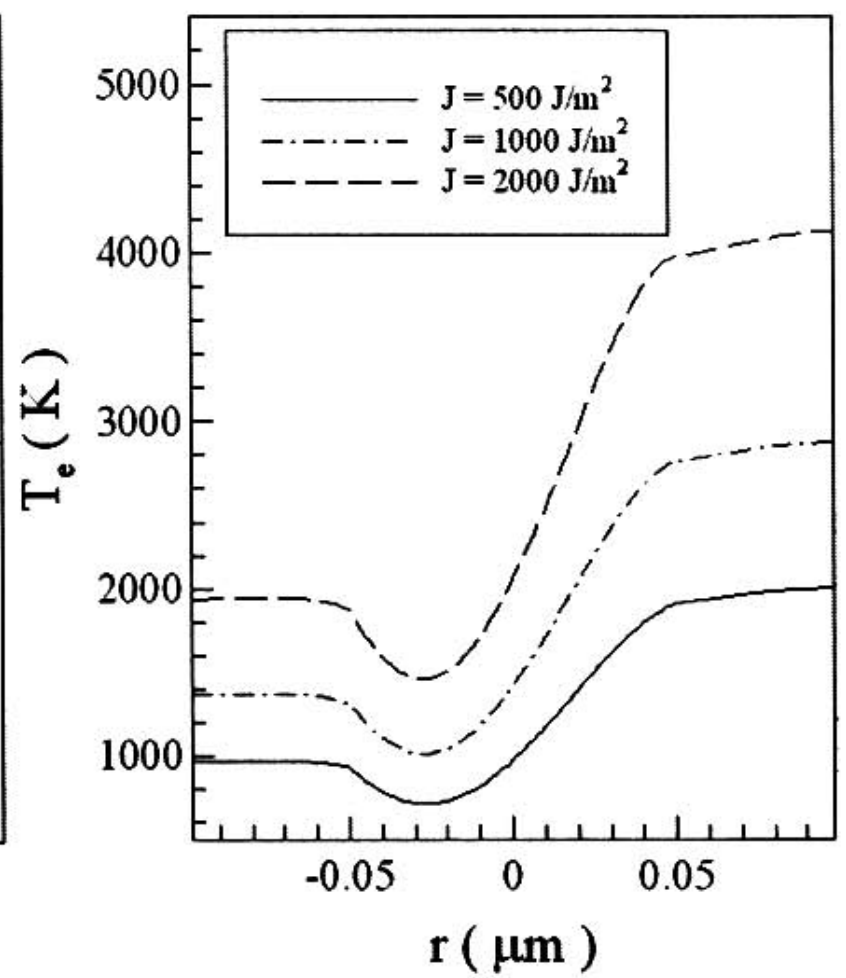

(b)

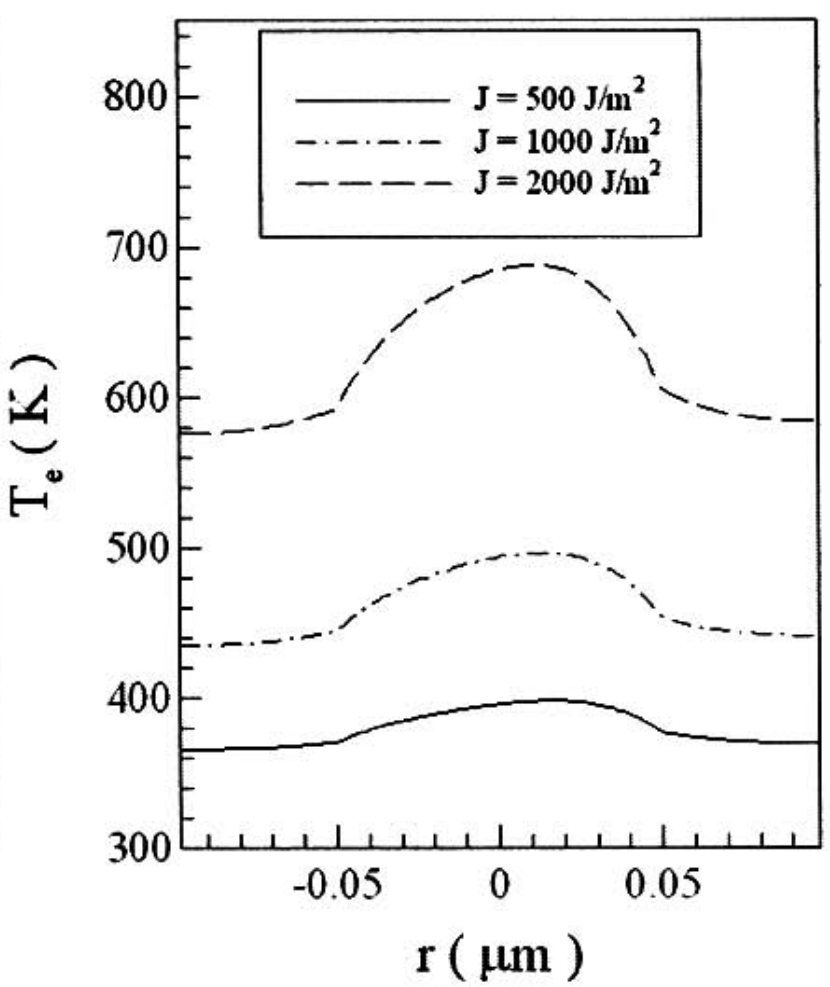

(d)

Fig. (13). Electron temperature profiles along the diameter at $\varphi=0$ and $\varphi=\pi$ at different times (a) $t=0.25 \mathrm{ps,} \mathrm{(b)} t=0.5 \mathrm{ps},(\mathbf{c}) t=$ $10 \mathrm{ps}$, and (d) $t=20 \mathrm{ps}$ with a mesh of $80 \times 20 \times 20$ and three different laser fluences $(J)$ of $500 \mathrm{~J} / \mathrm{m}^{2}, 1000 \mathrm{~J} / \mathrm{m}^{2}$ and $2000 \mathrm{~J} / \mathrm{m}^{2}$.

Fig. (15) shows the displacement $u_{r}$ along the diameter at $\varphi=0$ and $\varphi=\pi$ at different times (a) $\mathrm{t}=5 \mathrm{ps}$, (b) $\mathrm{t}=10 \mathrm{ps,}$ (c) $\mathrm{t}=15 \mathrm{ps}$, and (d) $\mathrm{t}=20$ ps with a mesh of
$80 \times 20 \times 20$ and three different laser fluences $\left(J=500 \mathrm{~J} / \mathrm{m}^{2}\right.$, $1000 \mathrm{~J} / \mathrm{m}^{2}$ and $2000 \mathrm{~J} / \mathrm{m}^{2}$ ). It can be seen that the displacement $u_{r}$ moves in the positive $r$ direction, implying the 


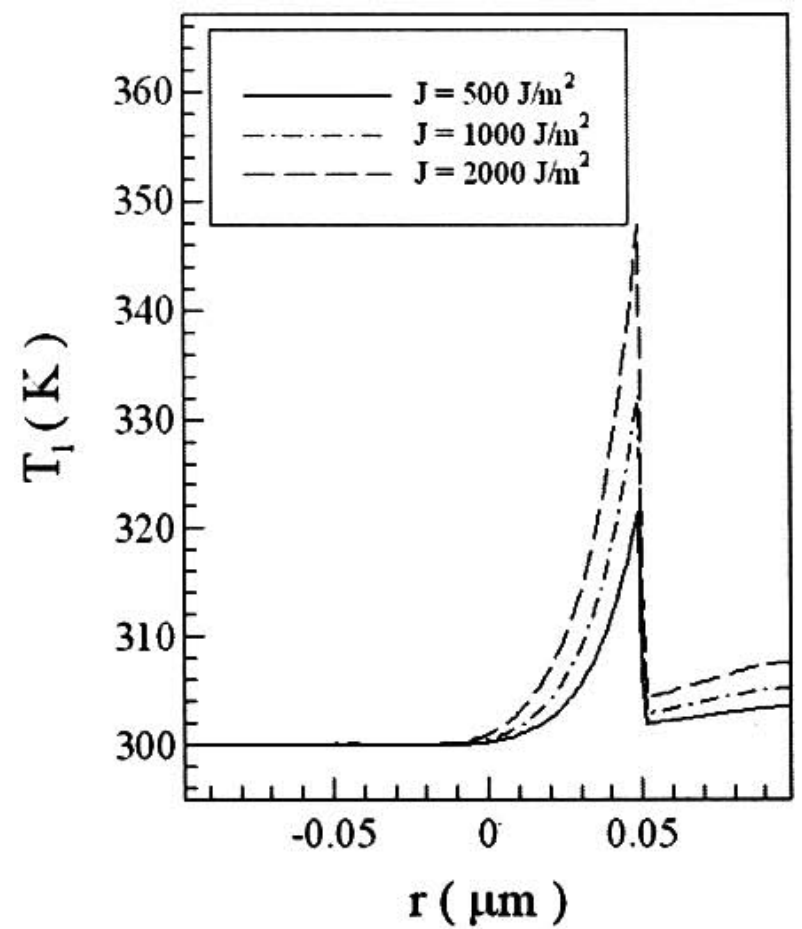

(a)

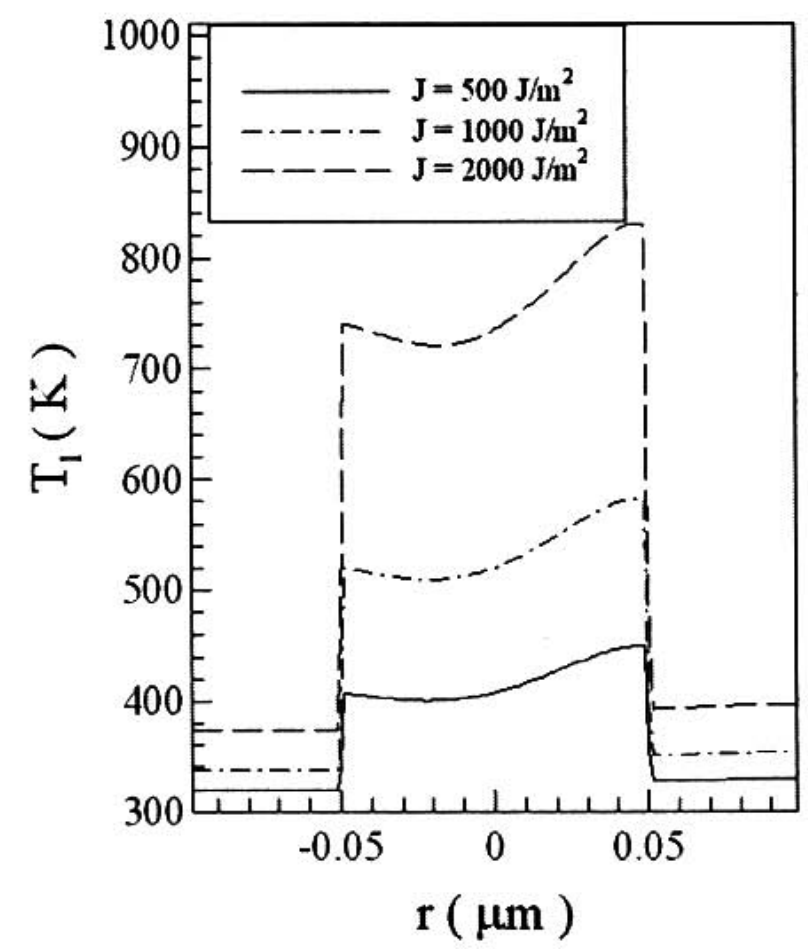

(c)

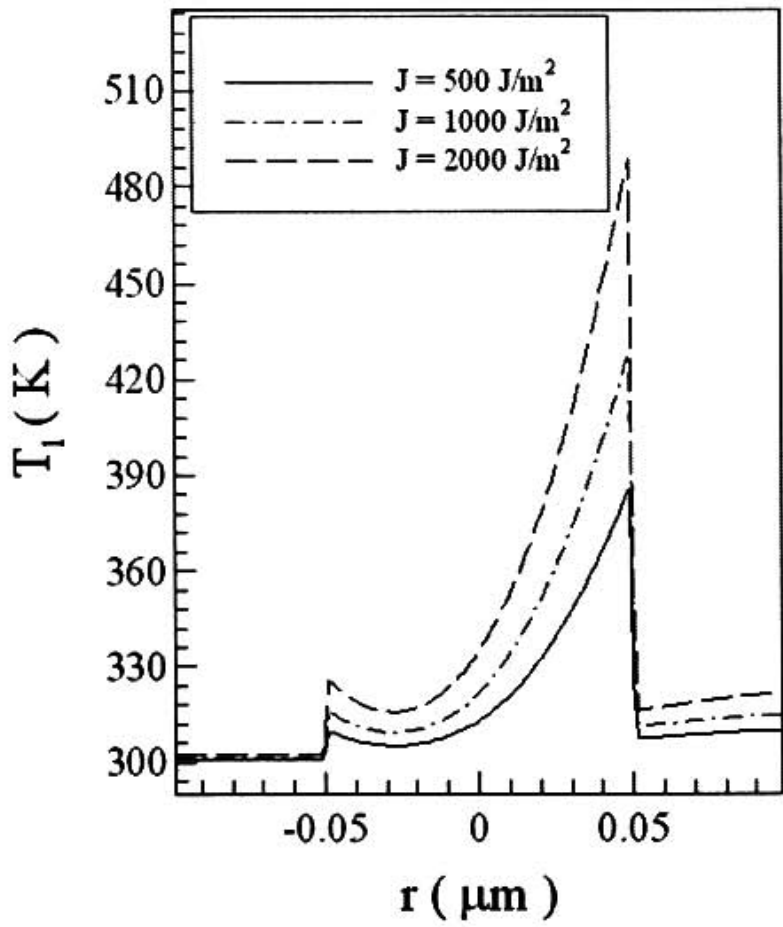

(b)

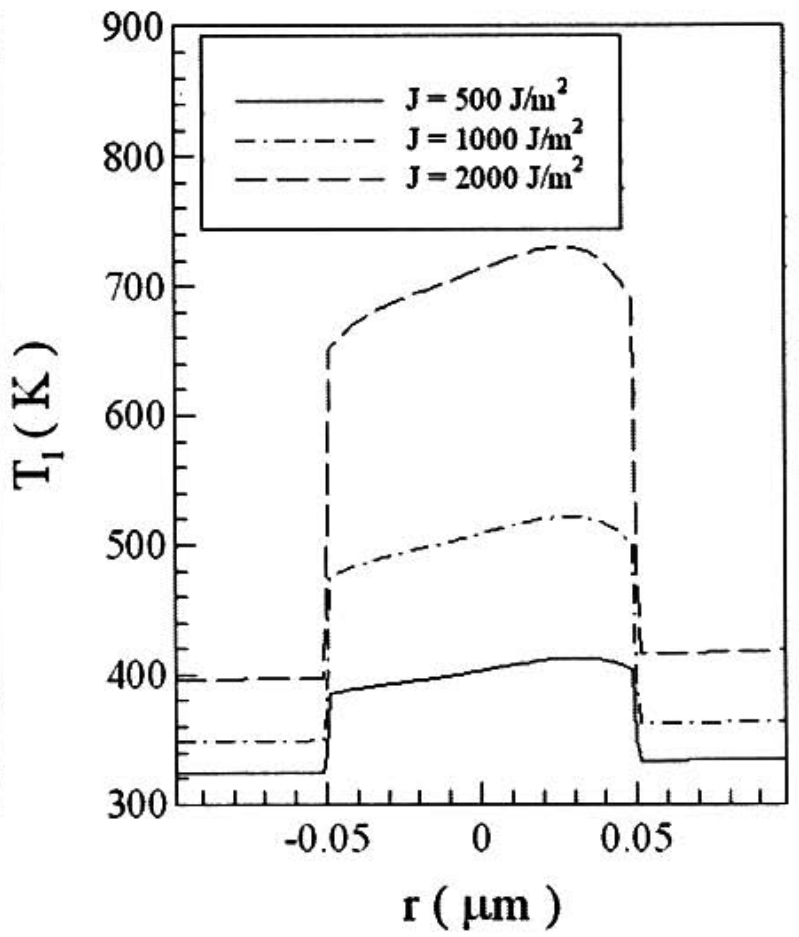

(d)

Fig. (14). Lattice temperature profiles along the diameter at $\varphi=0$ and $\varphi=\pi$ at different times (a) $t=0.25 \mathrm{ps,} \mathrm{(b)} t=0.5 \mathrm{ps},(\mathbf{c}) t=$ $10 \mathrm{ps}$, and (d) $t=20 \mathrm{ps}$ with a mesh of $80 \times 20 \times 20$ and three different laser fluences $(J)$ of $500 \mathrm{~J} / \mathrm{m}^{2}, 1000 \mathrm{~J} / \mathrm{m}^{2}$ and $2000 \mathrm{~J} / \mathrm{m}^{2}$.

sphere is expanding. However, the displacement gradient shows a clear difference across the interface, implying that the bond between these two layers could be damaged under high intensity laser irradiation.
Figs. (16) and (17) show contours of the electron temperature distribution and the lattice temperature distribution in the cross section of $\theta=0$ and $\theta=\pi$ at different times (a) $\mathrm{t}$ $=0.25 \mathrm{ps}$, (b) $\mathrm{t}=0.5 \mathrm{ps}$, (c) $\mathrm{t}=10 \mathrm{ps}$, and (d) $\mathrm{t}=20 \mathrm{ps}$, 


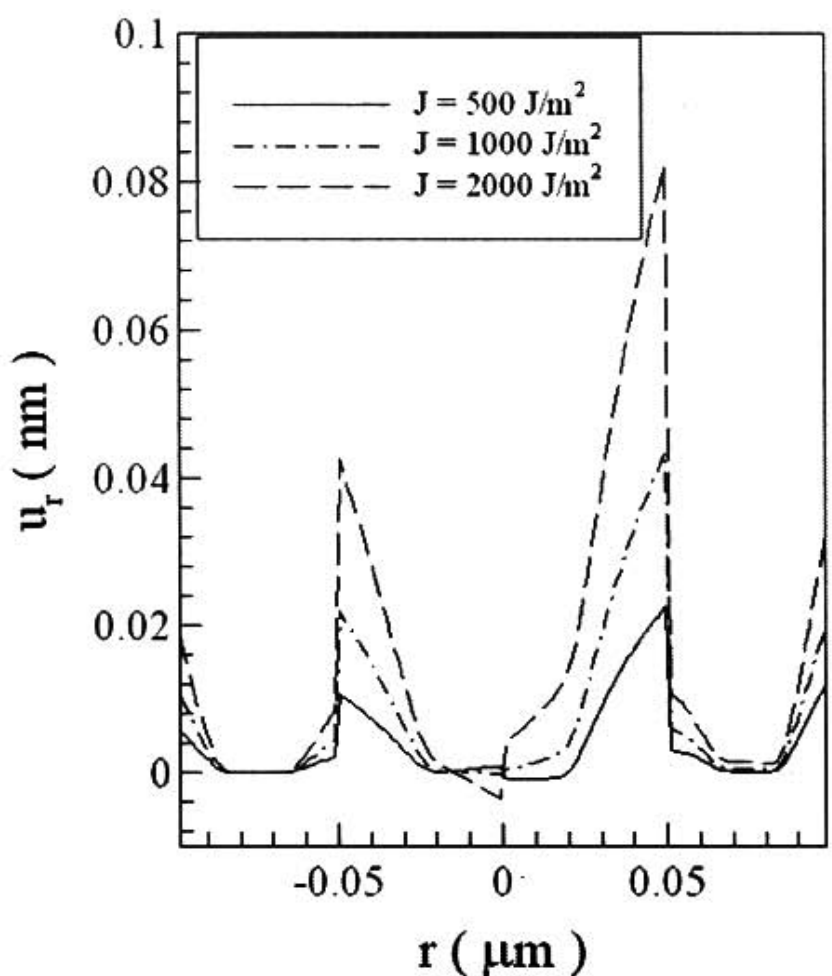

(a)

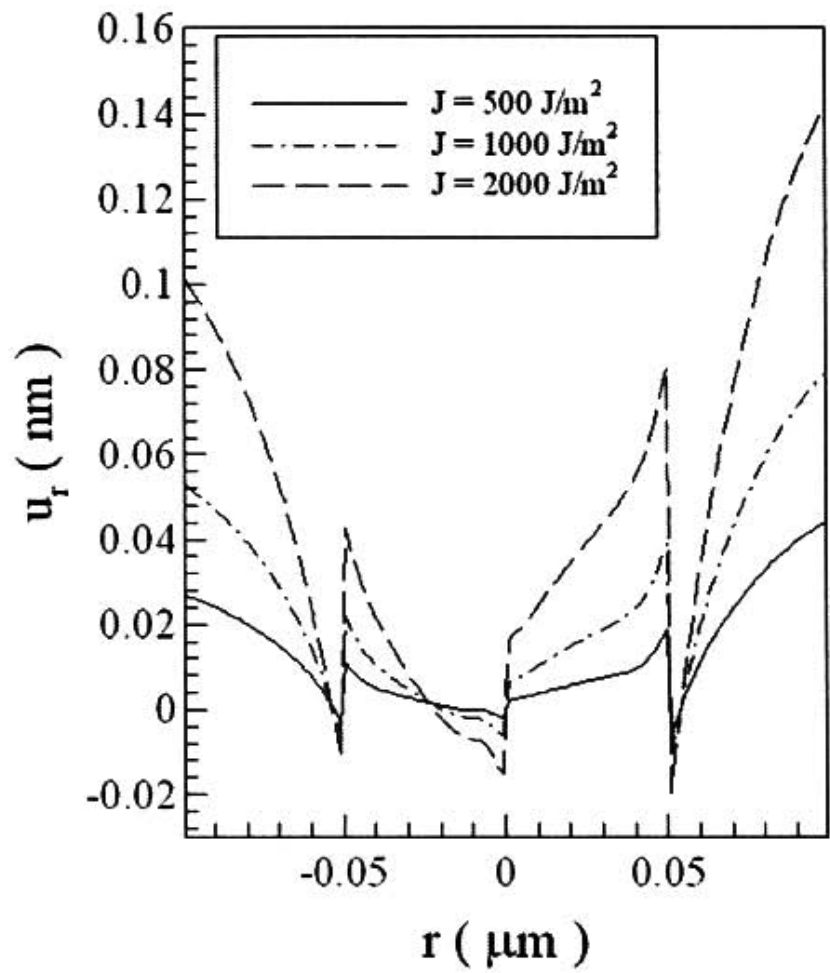

(c)

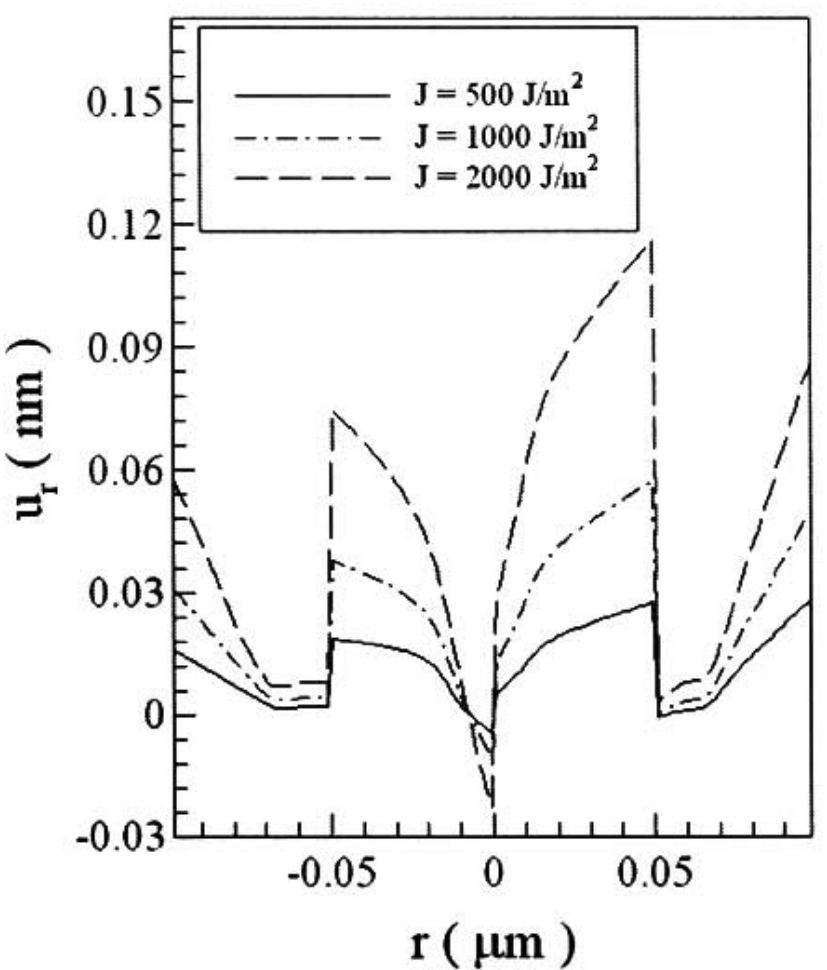

(b)

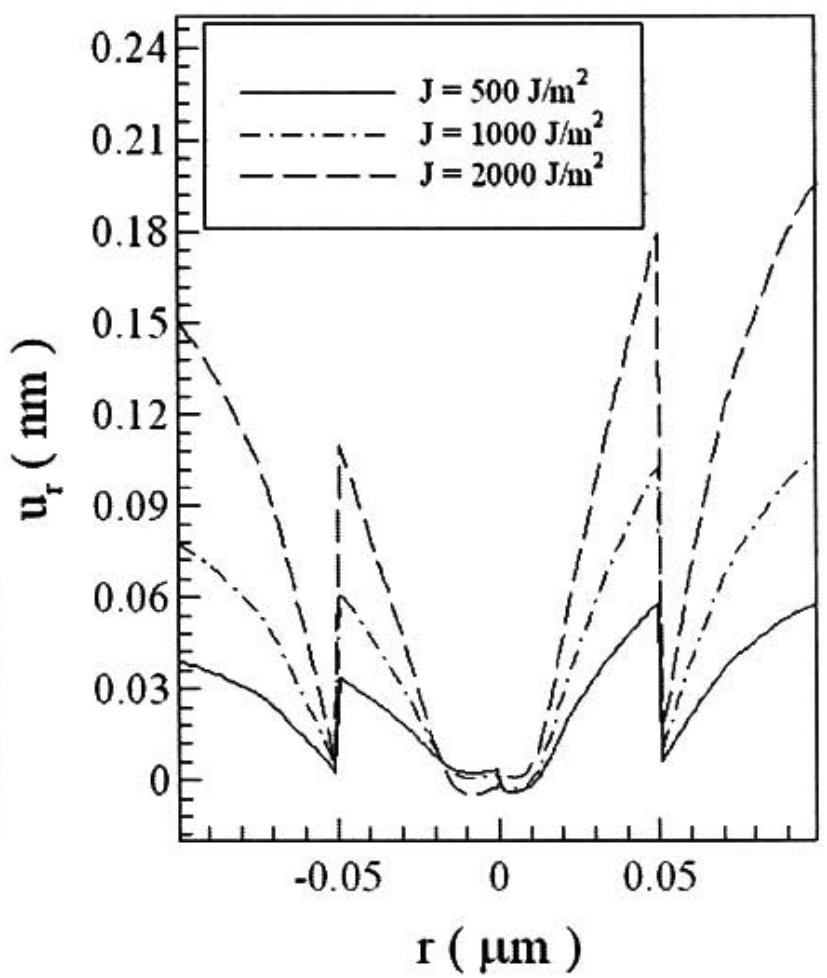

(d)

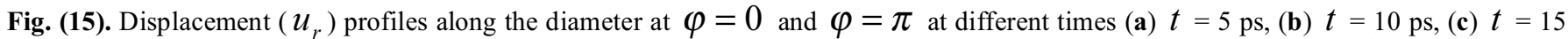
ps, and (d) $t=20$ ps with a mesh of $80 \times 20 \times 20$ and three different laser fluences $(J)$ of $500 \mathrm{~J} / \mathrm{m}^{2}, 1000 \mathrm{~J} / \mathrm{m}^{2}$ and $2000 \mathrm{~J} / \mathrm{m}^{2}$.

respectively. Again, both figures show that the heat is transferred from the upper hemisphere to the lower hemisphere and also from the gold layer to the chromium layer.
Fig. (18) shows contours of the displacement $u_{r}$ in the cross section of $\theta=0$ and $\theta=\pi$ at different times (a) $t=5$ ps, (b) $\mathrm{t}=10 \mathrm{ps}$, (c) $\mathrm{t}=15 \mathrm{ps}$, and (d) $\mathrm{t}=20 \mathrm{ps}$, respectively. It can be seen that the sphere is expanding and further the 


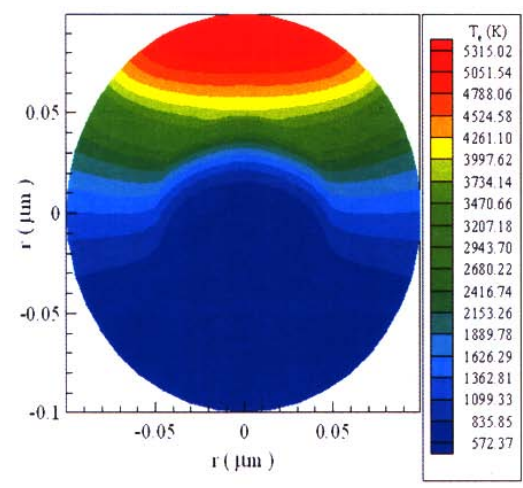

(a)

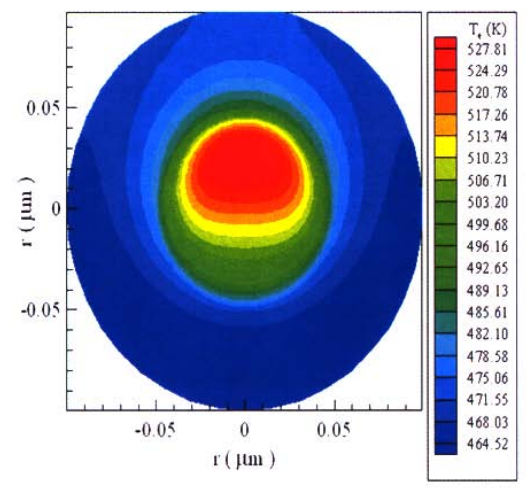

(c)

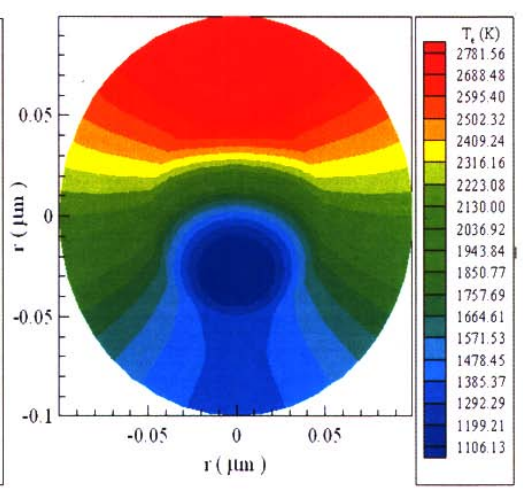

(b)

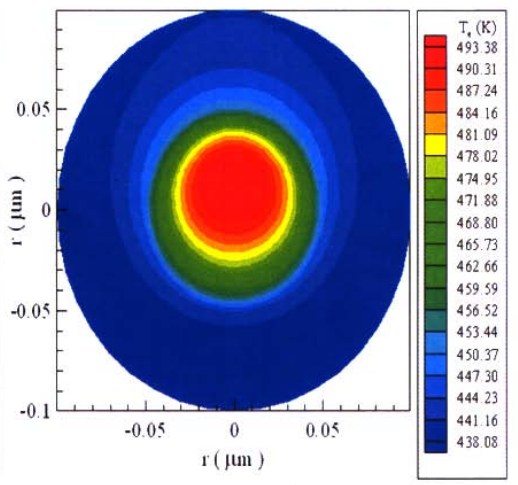

(d)

Fig. (16). Contours of electron temperature distributions in the cross section of $\theta=0$ and $\theta=\pi$ at different times (a) $t=0.25$ ps, (b) $t=$ $0.5 \mathrm{ps}$, (c) $t=10 \mathrm{ps}$, and (d) $t=20 \mathrm{ps}$ with a mesh of $80 \times 20 \times 20$ and a laser fluence $(J)$ of $1000 \mathrm{~J} / \mathrm{m}^{2}$.

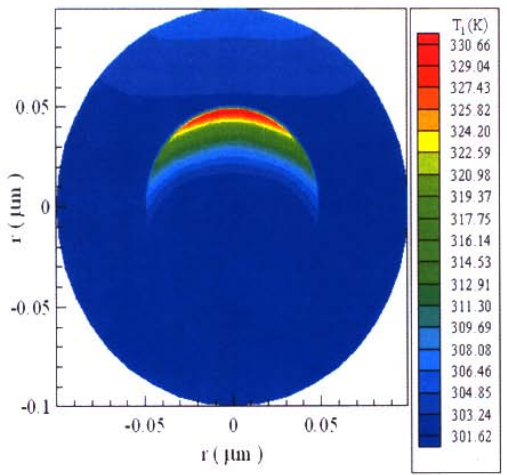

(a)

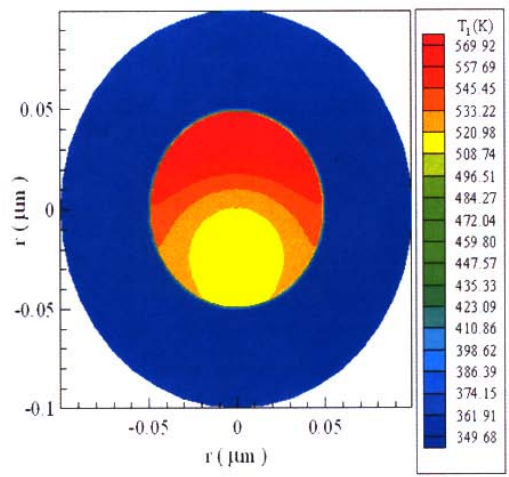

(c)

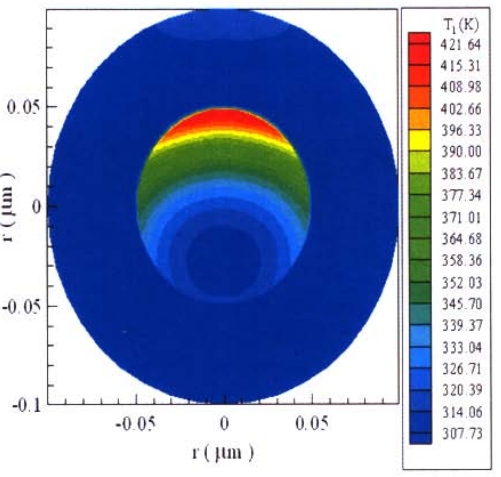

(b)

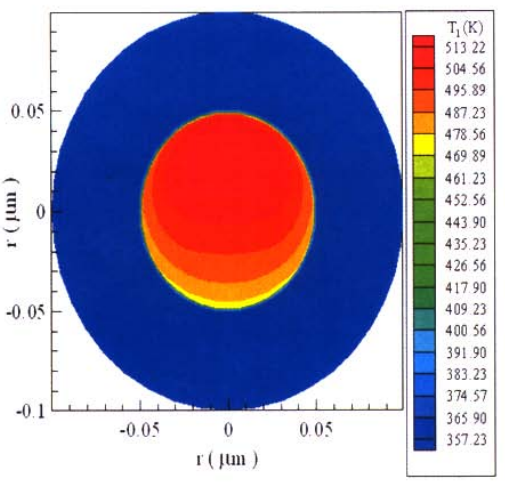

(d)

Fig. (17). Contours of lattice temperature distributions in the cross section of $\theta=0$ and $\theta=\pi$ at different times (a) $t=0.25 \mathrm{ps,}$, (b) $t=$ $0.5 \mathrm{ps},(\mathbf{c}) t=10 \mathrm{ps}$, and (d) $t=20 \mathrm{ps}$ with a mesh of $80 \times 20 \times 20$ and a laser fluence $(J)$ of $1000 \mathrm{~J} / \mathrm{m}^{2}$. 


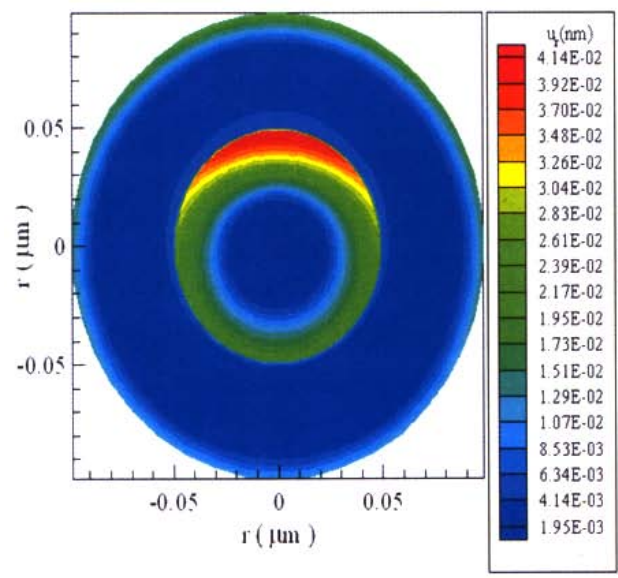

(a)

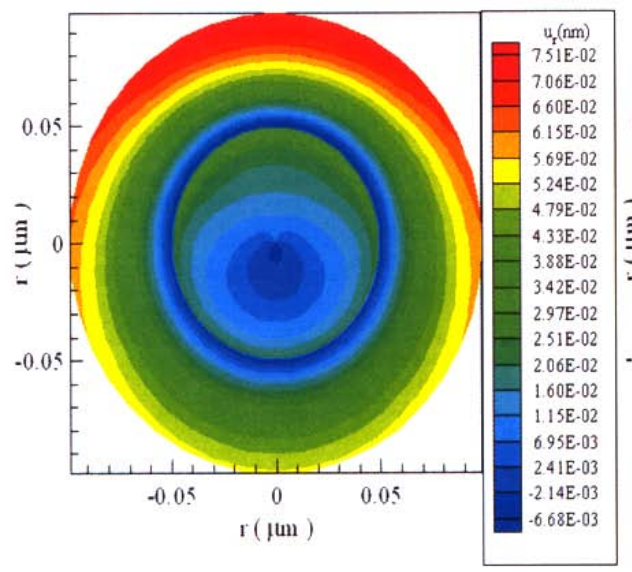

(c)

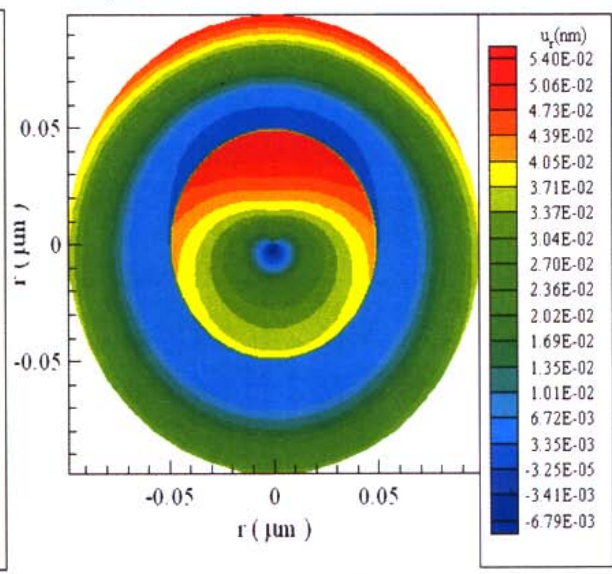

(b)

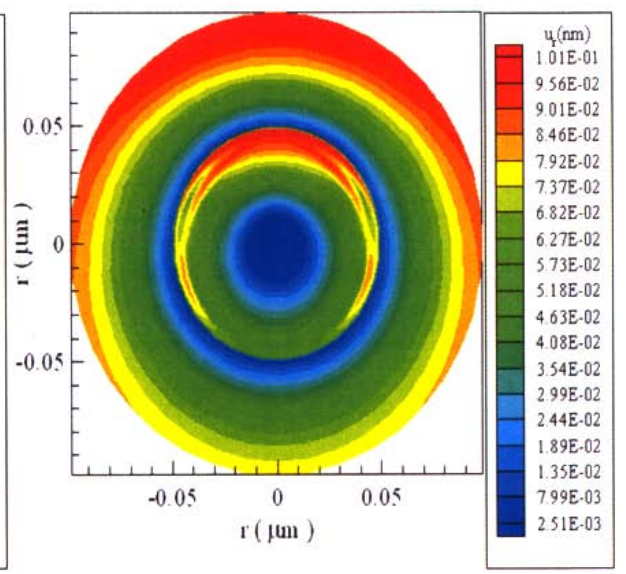

(d)

Fig. (18). Contours of displacement $\left(u_{r}\right)$ distributions in the cross section of $\theta=0$ and $\theta=\pi$ at different times (a) $t=5 \mathrm{ps,} \mathrm{(b)} t=10$ ps, (c) $t=15 \mathrm{ps}$, and (d) $t=20 \mathrm{ps}$ with a mesh of $80 \times 20 \times 20$ and a laser fluence $(J)$ of $1000 \mathrm{~J} / \mathrm{m}^{2}$.

upper hemisphere expands more than the lower hemisphere because the laser irradiates the upper hemisphere.

\section{CONCLUSION}

We have developed a finite difference method for studying thermal deformation in 3D double-layered thin films and micro spheres exposed to ultrashort-pulsed lasers. The method, based on the parabolic two-step heat transport equations, accounts for the coupling effect between lattice temperature and strain rate, as well as for the hot-electron blast effect in momentum transfer. By replacing the displacement components in the dynamic equations of motion using the velocity components, developing a fourth-order compact method for evaluating stress derivatives in the dynamic equations of motion, and employing a staggered grid, we have developed a numerical method that allows us to avoid non-physical oscillations in the solution. Numerical results show that the central part of the thin film and the micro sphere expand and the bond between these two layers could be damaged under high intensity laser irradiation.

\section{NOMENCLATURE}

$C_{e}, C_{l} \quad=\quad$ electron and lattice heat capacity, respectively

\begin{tabular}{|c|c|c|}
\hline$G$ & $=$ & electron - lattice coupling factor \\
\hline$J$ & $=$ & laser fluence \\
\hline$K$ & $=$ & bulk modulus \\
\hline$k_{e}$ & $=$ & thermal conductivity \\
\hline$L$ & $=$ & radius of sphere \\
\hline$L_{x} \times L_{y} \times L_{z}$ & $=$ & dimension of thin film \\
\hline$m$ & $=$ & index for layer \\
\hline$R$ & $=$ & surface reflectivity \\
\hline$Q$ & $=$ & energy absorption rate \\
\hline$(r, \theta, \varphi)$ & $=$ & spherical coordinates \\
\hline$r_{s}$ & $=$ & spatial profile parameter of laser \\
\hline$T_{e}, T_{l}$ & $=$ & $\begin{array}{l}\text { electron temperature and lattice } \\
\text { temperature }\end{array}$ \\
\hline$t, t_{n}$ & $=$ & time \\
\hline$t_{p}$ & $=$ & laser pulse duration \\
\hline$u, v, w$ & $=$ & $\begin{array}{l}\text { displacements in the } x, y \text { and } z \\
\text { directions, respectively }\end{array}$ \\
\hline$u_{r}, u_{\theta}, u_{\varphi}$ & $=$ & $\begin{array}{l}\text { displacements in the } r, \theta \text { and } \varphi \\
\text { directions, respectively }\end{array}$ \\
\hline
\end{tabular}




\begin{tabular}{|c|c|c|}
\hline$u_{i, j, k}^{n}$ & $=$ & $\begin{array}{l}\text { numerical solution of } \\
u\left(x_{i}, y_{j}, z_{k}, t_{n}\right)\end{array}$ \\
\hline$v_{1}, v_{2}, v_{3}$ & $=$ & $\begin{array}{l}\text { velocity components in the } x, \\
y \text { and } z \text { directions, respectively }\end{array}$ \\
\hline$(x, y, z)$ & $=$ & Cartesian coordinates \\
\hline$z_{s}, \zeta$ & $=$ & optical penetration depth \\
\hline$\alpha_{T}$ & $=$ & thermal expansion coefficient \\
\hline$\Delta t, \Delta x, \Delta y, \Delta z$ & $=$ & $\begin{array}{l}\text { time increment and spatial step } \\
\text { sizes, } \\
\text { respectively }\end{array}$ \\
\hline$\Delta_{-t}, \delta_{x}, \delta_{y}, \delta_{z}$ & $=$ & finite difference operators \\
\hline$\varepsilon_{x}, \varepsilon_{y}, \varepsilon_{z}$ & $=$ & $\begin{array}{l}\text { normal strains in the } x, y \text { and } z \\
\text { directions, respectively }\end{array}$ \\
\hline$\varepsilon_{r}, \varepsilon_{\theta}, \varepsilon_{\varphi}$ & $=$ & $\begin{array}{l}\text { normal strains in the } r, \theta \text { and } \varphi \\
\text { directions, respectively }\end{array}$ \\
\hline$\Lambda$ & $=$ & electron - blast coefficient \\
\hline$\gamma_{x y}, \gamma_{x z}, \gamma_{y z}$ & $=$ & $\begin{array}{l}\text { shear strains in Cartesian coordi- } \\
\text { nates }\end{array}$ \\
\hline$\varepsilon_{r \theta}, \varepsilon_{r \varphi}, \varepsilon_{\theta \varphi}$ & $=$ & $\begin{array}{l}\text { shear strains in spherical coordi- } \\
\text { nates }\end{array}$ \\
\hline$\lambda, \mu$ & $=$ & Lame's coefficients \\
\hline$\rho$ & $=$ & density \\
\hline$\sigma_{x}, \sigma_{y}, \sigma_{z}$ & $=$ & $\begin{array}{l}\text { normal stresses in the } x, y \text { and } \\
z \text { directions, respectively }\end{array}$ \\
\hline$\sigma_{r}, \sigma_{\theta}, \sigma_{\varphi}$ & $=$ & $\begin{array}{l}\text { normal stresses in the } r, \theta \text { and } \varphi \\
\text { directions, respectively }\end{array}$ \\
\hline$\sigma_{x y}, \sigma_{x z}, \sigma_{y z}$ & $=$ & $\begin{array}{l}\text { shear stresses in the } x, y \text { and } z \\
\text { directions, respectively }\end{array}$ \\
\hline$\sigma_{r \theta}, \sigma_{r \varphi}, \sigma_{\theta \varphi}$ & $=$ & $\begin{array}{l}\text { shear stresses in the } r, \theta \text { and } \varphi \\
\text { directions, respectively }\end{array}$ \\
\hline
\end{tabular}

\section{REFERENCES}

[1] Tzou DY, Chen JK, Beraun JE. Hot-electron blast induced by ultrashort-pulsed lasers in layered media. Int J Heat Mass Transfer 2002; 45: 3369-82.

[2] Wang H, Dai W, Nassar R, Melnik RVN. A finite difference method for studying thermal deformation in a thin film exposed to ultrashort-pulsed lasers. Int J Heat Mass Transfer 2006; 49: 2712-23.

[3] Wang H, Dai W, Melnik RVN. A finite difference method for studying thermal deformation in a double-layered thin film exposed to ultrashort-pulsed lasers. Int J Thermal Sci 2006; 46: 1179-96.

[4] Wang H, Dai W, Hewavitharana LG. A finite difference method for studying thermal deformation in a double-layered thin film with imperfect interfacial contact exposed to ultrashort-pulsed lasers. Int J Thermal Sci 2008; 47: 7-24.

[5] Chen JK, Beraun JE, Tham CL. Comparison of one-dimensional and two-dimensional axisymmetric approaches to the thermomechanical response caused by ultrashort laser heating. J Opt A Pure Appl Opt 2002; 4: 650-61.

[6] Chen JK, Beraun JE, Tham CL. Investigation of thermal response caused by pulse laserheating. Numer Heat Transfer Part A 2003; 44: 705-22

[7] Chen JK, Beraun JE, Tzou DY. Thermomechanical response of metal films heated by ultrashort-pulsed lasers. J Thermal Stresses 2002; 25: 539-58.

[8] Du X, Dai W, Wang P. A finite difference method for studying thermal deformation in a 3D micro-sphere exposed to ultrashort pulsed lasers. Numer. Heat Transfer Part A 2008; 53: 457-84.

[9] Zhang S, Dai W, Wang H, Melnik RVN. A finite difference method for studying thermal deformation in a $3 \mathrm{D}$ thin film exposed to ultrashort pulsed lasers. Int J Heat Mass Transfer 2008; 53: 457-84.

[10] Reismann H, Pawlik PS. Elasticity, theory and applications. New York: Wiley 1980.

[11] Qiu TQ, Tien CL. Short-pulse laser heating on metals. Int J Heat Mass Transfer 1992; 35: 719-26.

[12] Tzou DY. Macro to micro heat transfer, the lagging behavior. Washington DC: Taylor \& Francis 1997.

[13] Qiu TQ, Tien CL. Femtosecond laser heating of multi-layer metals -I. analysis. Int J Heat Mass Transfer 1994; 37: 2789-97.

[14] Sadd MH. Elasticity: theory, applications, and numerics. New York: Elsevier 2005.

[15] Kaba I, Dai W. A stable three-level finite difference scheme for solving the parabolic two-step model in a 3D micro-sphere heated by ultrashort-pulsed lasers. J Comput Appl Math 2005; 181: 12547.

[16] Smith GD. Numerical solution of partial differential equationsfinite difference methods. Oxford: Clarendon Press 1999.

[17] Kaye GWC. Tables of physical and chemical constants and some mathematical functions, 14th edn. London UK: Longman 1973. 